\title{
Discretisations of higher order and the theorems of Faà di Bruno and DeMoivre-Laplace
}

\author{
IMME VAN DEN BERG
}

\begin{abstract}
We study discrete functions on equidistant and non-equidistant infinitesimal grids. We consider their difference quotients of higher order and give conditions for their near-equality to the corresponding derivatives. Important tools are nonstandard notions of regularity of higher order, and the formula of Faà di Bruno for higher order derivatives and a discrete version of it. As an application of such transitions from the discrete to the continuous we extend the DeMoivre-Laplace Theorem to higher order: $n$-th order difference quotients of the binomial probability distribution tend to the corresponding $n$-th order partial differential quotients of the Gaussian distribution.
\end{abstract}

2010 Mathematics Subject Classification 03H05, 39A10, 39A12, 60F05 (primary)

Keywords: Difference quotients, chain rule, Faà di Bruno Theorem, DeMoivreLaplace Theorem, nonstandard analysis

\section{Introduction}

Let $\delta \xi>0$ be infinitesimal in the sense of nonstandard analysis. We let $\mathbb{X}$ be the set of all multiples of $\delta \xi$ by some integer. We study discrete "quasi-continuous" functions $f$ on this set of equally spaced points, but also on more irregular grids, which are images of $\mathbb{X}$ by some near-standard function, say $\phi$. Such grids will be called near-continua.

Obviously, the well-known nonstandard notions of $S$-continuity and $S$-differentiability can be defined for near-continua, and we will extend these notions to regular behavior of all standard orders. This will lead to the notion of functions "of class $S^{n}$ ". This notion deals with the regularity of higher-order difference quotients much as the notion of functions of class $C^{n}$ deals with the regularity (here also existence) of higher-order derivatives.

We show that on equidistant near-continua $\mathbb{X}$ the difference quotients $\frac{\delta^{k} f}{\delta \xi^{k}}$ of functions of class $S^{n}$ are for $k \leq n$ nearly equal to the derivatives $\frac{d^{k \circ} f}{d x^{k}}$ of the shadow ${ }^{\circ} f$ of 
the function $f$ : the shadow of $f$ being the unique standard real function (if it exists) infinitely close to $f$ on the limited domain.

For functions defined on non-equidistant near-continua $\phi(\mathbb{X})$ such nearequality does not need to hold - even for functions as elementary as quadratic functions - and appears to depend on the nature of the function $\phi$. As is to be expected the Chain Rule will have some importance, in particular its version for higher order derivatives, known as the Formula of Faà di Bruno. We establish an approximate, discrete version of this formula. With the help of this formula we show that the difference quotients of standard order $n$ of a function $\psi: \phi(\mathbb{X}) \rightarrow \mathbb{R}$ are infinitely close to the corresponding derivatives of its shadow, provided that $\phi$ and $\psi$ are both of class $S^{n}$.

The present article extends the results on transitions between the discrete and the continuous on equidistant near-continua of [4], [11] and [6] to transitions on general near-continua. These studies ([11] representing a Masters Thesis supervised by the author) apply such results to the transition of the discrete binomial probability distribution to the continuous Gaussian distribution, extending the DeMoivre-Laplace theorem in a sense to difference quotients and (partial) derivatives of higher order. We end the present article by a short proof of this extension.

The article has the following structure. Nonstandard analysis disposes of a terminology common to both a class of discrete functions and a class of continuous functions; this terminology facilitates the transition between discreteness and continuity and will be recalled in Section 2. In Section 3 we define the notion of a function of class $S^{n}$ for general near-continua. We show how these functions behave for discrete differentiation and integration. Also, the class $S^{n}$ is stable for algebraic operations on equidistant near-continua, but we provide an elementary counterexample for non-equidistant nearcontinua. Later on, at the end of Section 5, conditions will be given for algebraic operations to hold on (suitable parts of) such near-continua. In Section 4 we recall the tools for the transition from discreteness to continuity (the class of standard functions of class $C^{n}$ ) at arbitrary order on equidistant near-continua. In Section 5 we develop the tools for the transition from discreteness to continuity at arbitrary order on general near-continua. It is here that we derive a nonstandard, discrete and approximative version of the formula of Faà di Bruno. In Section 6 we extend the process of continuisation to functions of two variables.

As an application of a continuisation at higher order we consider in Section 7 a higher order version of the DeMoivre-Laplace theorem. We introduce first (Subsection 7.1) a convenient rescaling for the Pascal triangle and the binomial coefficients, leading to the notion of the binomial function $b(t, x)$. The rescaling of the Pascal triangle results 
into the union of two grids equally spaced in time and in space; however the time-steps and the space-steps are not identical. The higher order DeMoivre-Laplace theorem in question states that on both grids the difference quotients of the binomial function are infinitely close to the corresponding partial derivatives of the Gaussian function, to all standard orders and for all limited non-infinitesimal $t$ and all limited $x$.

In Subsection 7.2 we establish a first-order difference equation for the binomial function and show that the function satisfies also a discrete heat equation. It appears to be convenient to extend this second-order partial difference equation to all orders. In the final Subsection 7.3 we complete the proof of the higher order DeMoivre-Laplace theorem, using the material on the transition from the discrete to the continuous on equidistant grids developed in earlier sections.

The article is written in the nonstandard axiomatic system IST of Nelson. A major difference to Robinson's nonstandard analysis [16] is the existence of nonstandard elements within infinite standard sets. Introductions to IST are for example contained in [14], [9], [7], and [8]. A thorough introduction to discrete probability theory in a setting similar to ours is contained in [15]. This theory is developed in an axiomatics which is weaker than $I S T$, essentially only the existence of nonstandard natural numbers and the Principle of External Induction over the standard natural numbers is needed. In fact this weak axiomatics is sufficient to develop the theory of discrete functions of class $S^{n}$ presented in the Sections 2 and 3. However, to establish the relation with classical continuity a substantial part of $I S T$ is needed, for one uses the notion of standard part or shadow of real numbers of functions. Their existence is given by the Standardization Axiom, and the Principle of Transfer is used, say, to be able to deal appropriately with standard functions for nonstandard arguments.

For a classical proof of the DeMoivre-Laplace theorem, see for instance [10].

\section{Functions of class $S^{0}$ and near-continua}

Functions of class $S^{0}$ have been introduced by F. Diener in [9], see also [8]. We recall here some basic properties. They are closely related to functions of class $C^{0}$.

We start by recalling the notion of $S$-continuity. This property is shared by the class of standard continuous functions and also a class of nonstandard discrete functions. As such the property is important for the interplay between discreteness and continuity. 
Definition 2.1 Let $A \subset \mathbb{R}$ and $f: A \rightarrow \mathbb{R}$. Let $x \in A$. The function $f$ is said to be $S$-continuous at $x$ if for all $y \in A$

$$
x \simeq y \Rightarrow f(x) \simeq f(y) .
$$

The function $f$ is said to be $S$-continuous on $A$ if $f$ is $S$-continuous at all $x \in A$.

Definition 2.2 Let $A \subset \mathbb{R}$ and $f: A \rightarrow \mathbb{R}$. The function $f$ is said to be of class $S^{0}$ on $A$ if $f(x)$ is limited and $S$-continuous at all limited $x \in A$.

A class of obvious examples of functions of class $S^{0}$ are the standard everywhere continuous functions.

By no means do functions of class $S^{0}$ need to be limited for unlimited $x$ : standard unbounded functions such as standard polynomials, as well as the exponential function, are all of class $S^{0}$. Standard rational functions with poles, like $f(x)=\frac{1}{1+x}$ are of class $S^{0}$ on all sets $A$ which do not contain elements infinitely close to the poles.

The next simple proposition often permits to verify that nonstandard functions are of class $S^{0}$.

Proposition 2.3 Let $A \subset \mathbb{R}$ and $f, g: A \rightarrow \mathbb{R}$. If $f$ is of class $S^{0}$ and $f(x) \simeq g(x)$ for all limited $x \in A$, then $g$ is also of class $S^{0}$.

Proof Clearly $g(x)$ is limited for all limited $x \in A$. Let $x \in A$ be limited and $y \in A, y \simeq x$. Then

$$
g(y) \simeq f(y) \simeq f(x) \simeq g(x)
$$

Hence $g$ is $S$-continuous at $x$.

In this article we consider for most of the time functions defined on a discrete subset of $\mathbb{R}$, consisting of successive points at an infinitesimal distance.

Definition 2.4 We let $\delta \xi$ always be a positive non-zero infinitesimal and $\mathbb{X}=\{k \delta \xi \mid k \in \mathbb{Z}\}$. The set $\mathbb{X}$ is called an equidistant near-continuum. Let $\mathbb{Y} \subset \mathbb{R}$ be internal. The set $\mathbb{Y}$ is called a near-continuum if it is the image of an equidistant near-continuum by a strictly monotone function $\phi: \mathbb{X} \rightarrow \mathbb{R}$ of class $S^{0}$.

For convenience we suppose, unless otherwise said, that $\phi$ is increasing, unbounded from below and unbounded from above. 
Definition 2.5 Let $\mathbb{Y}=\phi(\mathbb{X})$ be a near-continuum, where $\phi: \mathbb{X} \rightarrow \mathbb{R}$ is a strictly increasing function of class $S^{0}$. Let $a, b \in \mathbb{Y}$ with $a<b$. We write

$$
[a \cdot b] \equiv\{\eta \in \mathbb{Y} \mid a \leq \eta \leq b\}
$$

and

$$
[a \cdot b[\equiv\{\eta \in \mathbb{Y} \mid a \leq \eta<b\} .
$$

Let $\alpha, \beta \in \mathbb{X}$ be such that $a=\phi(\alpha)$ and $b=\phi(\beta)$. The sets $[a \cdots b]$ and $[a \cdots b[$ are called a near-interval if $\alpha$ and $\beta$ are limited. Note that then also $a$ and $b$ are limited, and that the difference between two succesive points $\delta \phi(\xi) \equiv \phi(\xi+\delta \xi)-\phi(\xi)$ is always infinitesimal.

Let $\eta \in \mathbb{Y}$ and let $\xi \in \mathbb{X}$ be such that $\eta=\phi(\xi)$. Sometimes we write $\eta_{\xi}=\phi(\xi)$ and $\delta \eta_{\xi}=\delta \phi(\xi)$, and we may even write with abuse of notation $\delta \eta$ instead of $\delta \eta_{\xi}$; note that generally speaking $\delta \eta$ is not a constant. Given a subset $A \subseteq \mathbb{Y}$ we define

$$
A^{(n)}=\left\{\eta_{\xi} \in A \mid(\forall i)\left(0 \leq i \leq n \Rightarrow \eta_{\xi+i \delta \xi} \in A\right)\right\}
$$

Notice that if $f$ is a real function defined on $A \subseteq \mathbb{X}$, the $n^{\text {th }}$-order difference quotient or $n^{\text {th }}$-order discrete derivative of $f$

$$
\frac{\delta^{n} f}{\delta \xi^{n}}(\xi) \equiv \frac{1}{\delta \xi^{n}} \sum_{j=0}^{n}(-1)^{j}\left(\begin{array}{l}
n \\
j
\end{array}\right) f(\xi+(n-j) \delta \xi)
$$

is defined on $A^{(n)}$, provided $A^{(n)} \neq \varnothing$.

We give two examples of functions of class $S^{0}$ defined on an equidistant near-continuum $\mathbb{X} \equiv\{k \delta \xi \mid k \in \mathbb{Z}\}$. The nonstandard function $\mathcal{E}: \mathbb{X} \rightarrow \mathbb{R}$ defined by

$$
\mathcal{E}(\xi)=(1+\delta \xi)^{\frac{\xi}{\delta \xi}}
$$

is limited and $S$-continuous for all limited $x$. Indeed, one proves easily that the Euler formula $\mathcal{E}(\xi) \simeq e^{\xi}$ holds for all limited $\xi \in \mathbb{X}$, and then one may apply Proposition 2.3. A second nonstandard example is the function $\mathcal{F}$ defined on $\mathbb{X}^{+} \equiv\{x \in \mathbb{X} \mid x \geq 0\}$ by

$$
\mathcal{F}(\xi)=\prod_{0 \leq \eta<\xi}(1+\eta \delta \xi)
$$

Then it is easy to verify that $\mathcal{F}(\xi) \simeq e^{\xi^{2} / 2}$ for all limited $\xi \in \mathbb{X}^{+}$. Again it follows from Proposition 2.3 that $\mathcal{F}$ is of class $S^{0}$ on $\mathbb{X}^{+}$. The discrete functions $\mathcal{E}$ and $\mathcal{F}$ have even more regularity, as will be shown in Section 3.

The class of functions of class $S^{0}$ is closed under the usual algebraic operations, provided one does not divide by infinitesimal values. The proof of the next proposition is straightforward. 
Proposition 2.6 Let $A \subset \mathbb{R}$ and $f, g: A \rightarrow \mathbb{R}$ be functions of class $S^{0}$. Then $-f, f+g$ and $f \cdot g$ are of class $S^{0}$, and $f / g$ is of class $S^{0}$ on all sets $B \subset A$ such that $g \neq 0$ on the limited part of $B$.

To functions of class $S^{0}$, defined on a possibly discrete subset of $\mathbb{R}$, may be associated standard continuous functions. This transition from the discrete to the continuous, or continuisation, uses the notion of shadow.

Any limited number $y$ is nearly-equal to a unique standard number $x$ called the standard part or the shadow of $y$, and we write $x={ }^{\circ} y$. For instance, if $\epsilon \simeq 0$, one has ${ }^{\circ} \epsilon=0$ and ${ }^{\circ} e^{\epsilon}=1$. To each function $f$ of class $S^{0}$ defined on $\mathbb{R}$ one may associate a unique standard function ${ }^{\circ} f$ such that $f(x) \simeq{ }^{\circ} f(x)$ for all limited $x$. The function ${ }^{\circ} f$ is called the standard part or shadow of $f$. In addition, the function ${ }^{\circ} f$ is continuous. This property was already known to Robinson [16]. We state a version of this theorem for discrete functions on a near-continuum.

Theorem 2.7 (Theorem of the continuous shadow, one variable) Let $\mathbb{Y}$ be a nearcontinuum. Let $f: \mathbb{Y} \rightarrow \mathbb{R}$ be of class $S^{0}$. Then there exists a unique standard function ${ }^{\circ} f: \mathbb{R} \rightarrow \mathbb{R}$ such that $f(y) \simeq{ }^{\circ} f(y)$ for all limited $y \in \mathbb{Y}$. In fact $f$ is everywhere continuous.

It is this theorem which yields a general procedure for the transformation of discrete functions into continuous functions. For a proof of the theorem we refer to the literature [16][14][8].

\section{Functions of class $S^{n}$ of one variable}

We extend the notion of functions of class $S^{0}$ to functions of class $S^{n}$ for all standard $n \in \mathbb{N}$. Functions of class $S^{n}$ are not only limited and $S$-continuous themselves, but also their difference quotients of order $m$ for $m \leq n$. We show that discrete derivation and integration relate functions of class $S^{n}$ and $S^{n+1}$ in a similar way as ordinary derivation

and integration relate functions of class $C^{n}$ and $C^{n+1}$. Stability of the (external) set of functions of class $S^{n}$ appears to hold for all the usual algebraic operations on equidistant near-continua. On general near-continua stability may depend on the nature of the near-continuum. Hence on equidistant near-continua polynomials of standard degree and limited coefficients are of class $S^{n}$, and rational functions which are quotients of such polynomials are of class $S^{n}$ whenever the arguments are not infinitely close to a singularity of the denominator, while such properties may not hold on all near-continua.

Journal of Logic \& Analysis 5:6 (2013) 
Notation 3.1 We let $\mathbb{Y}=\phi(\mathbb{X})$ be a near-continuum, where $\phi: \mathbb{X} \rightarrow \mathbb{R}$ is a strictly increasing function of class $S^{0}$. Let $\psi: \mathbb{Y} \rightarrow \mathbb{R}$. Let $\eta \in \mathbb{Y}$ and let $\xi \in \mathbb{X}$ be such that $\eta=\phi(\xi)$. Depending on the context we use the following notations for the differences of $\psi$ :

$$
\delta \psi(\eta)=\delta \psi\left(\eta_{\xi}\right)=\psi\left(\eta_{\xi}+\delta \eta_{\xi}\right)-\psi\left(\eta_{\xi}\right)=\psi(\phi(\xi+\delta \xi))-\psi(\phi(\xi)) .
$$

We write $\psi^{[0]}=\psi$. Let $n \in \mathbb{N}, n \geq 1$. Assume $\psi^{[n-1]}(\eta)$ is defined. Again depending on the context we define

$$
\begin{aligned}
\psi^{[n]}(\eta) & \equiv \frac{\delta \psi^{[n-1]}}{\delta \eta}(\eta)=\frac{\delta \psi^{[n-1]}}{\delta \eta_{\xi}}\left(\eta_{\xi}\right)=\frac{\psi^{[n-1]}\left(\eta_{\xi}+\delta \eta_{\xi}\right)-\psi^{[n-1]}\left(\eta_{\xi}\right)}{\delta \eta_{\xi}} \\
& =\frac{\psi^{[n-1]}(\phi(\xi+\delta \xi))-\psi(\phi(\xi))}{\phi(\xi+\delta \xi)-\phi(\xi)} .
\end{aligned}
$$

Observe that, if $\psi$ is defined on $A \subseteq \mathbb{Y}$ and $A^{(n)} \neq \varnothing$, its $n^{\text {th }}$-order difference quotient $\psi^{[n]}$ is defined on $A^{(n)}$.

A real function is of class $C^{n}$ if it is $n$ times continuously differentiable. We define functions of class $S^{n}$ by analogy.

Definition 3.2 Let $A \subseteq \mathbb{Y}$ and $\psi: A \rightarrow \mathbb{R}$, and let $n \in \mathbb{N}, n \geq 1$ be standard. Assume $A^{(n)} \neq \varnothing$. We say that $\psi$ is of class $S^{n}$ on $A$ if $\psi^{[k]}: A^{(k)} \rightarrow \mathbb{R}$ is of class $S^{0}$ for all $k$ with $0 \leq k \leq n$.

The next proposition is a first consequence of Definition 3.2.

Proposition 3.3 Let $A \subset \mathbb{Y}$ and let $n \in \mathbb{N}, n \geq 1$ be standard. Assume $A^{(n)} \neq \varnothing$. Let $\psi: A \rightarrow \mathbb{R}$ be of class $S^{n}$ on $A$ and assume $0 \leq m<n$. Then $\psi$ is of class $S^{m}$ on $A$.

We now show that the difference quotient $\psi^{[1]}$ of a function $\psi$ of class $S^{n}$ is of class $S^{n-1}$, implying that the difference quotient $\psi^{[m]}$ of order $m<n$ is a function of class $S^{n-m}$. Conversely, if the difference quotient of a function $\psi$ of class $S^{0}$ is of class $S^{n-1}$, the function $\psi$ will be of class $S^{n}$.

For convenience we consider functions defined on the whole of $\mathbb{Y}$.

Lemma 3.4 (Lemma of the discrete derivative) Let $n \in \mathbb{N}$ be standard and $\psi: \mathbb{Y} \rightarrow \mathbb{R}$ be of class $S^{n}$. Then $\psi^{[1]}$ is of class $S^{n-1}$.

Proof Let $\psi$ be a function of class $S^{n}$. Then $\left(\psi^{[1]}\right)^{[k]}=\psi^{[k+1]}$ is of class $S^{0}$ for all $k$ with $0 \leq k \leq n-1$. Hence $\psi^{[1]}$ is of class $S^{n-1}$. 
Proposition 3.5 Let $m, n \in \mathbb{N}$ be standard with $m \leq n$ and let $\psi: \mathbb{Y} \rightarrow \mathbb{R}$ be of class $S^{n}$. Then $\psi^{[m]}$ is a function of class $S^{n-m}$.

Next lemma bears some relation to the following well-known theorem relating the convergence of sequences of functions and their derivatives: Given a sequence of real functions $\left(f_{n}\right)_{n \in \mathbb{N}}$, if the sequence of derivatives $\left(f_{n}^{\prime}\right)_{n \in \mathbb{N}}$ converges uniformly to a real function $g$, and if at some point $x_{0}$ the sequence $\left(f_{n}\left(x_{0}\right)\right)_{n \in \mathbb{N}}$ converges, the functions $\left(f_{n}\right)_{n \in \mathbb{N}}$ converge uniformly to a function $f$ with $f^{\prime}=g$.

Lemma 3.6 (Lemma of the discrete integral) Let $n \in \mathbb{N}$ be standard and $\psi: \mathbb{Y} \rightarrow \mathbb{R}$. If $\psi^{[1]}$ is of class $S^{n}$ and $\psi\left(y_{0}\right)$ is limited for some limited $y_{0} \in \mathbb{Y}$, then $\psi$ is of class $S^{n+1}$.

Proof Let $\psi^{[1]}$ be of class $S^{n}$. Then $\psi^{[k]}=\left(\psi^{[1]}\right)^{[k-1]}$ is of class $S^{0}$ for all $k$ with $1 \leq k \leq n+1$. We prove that also $\psi$ is of class $S^{0}$. Let $z \in \mathbb{Y}$ be limited, with $y_{0}<z$. Now $\psi^{[1]}(\eta)$ is limited for all $\eta$ with $y_{0} \leq \eta<z$, so $\psi(z)=\psi\left(y_{0}\right)+$ $\sum_{y_{0} \leq \eta<z} \psi^{[1]}(\eta) \delta y$ is limited. If $z<y_{0}$, the proof that $\psi(z)$ is limited is analogous. Let $y, z \in \mathbb{Y}$ be limited, with $y \simeq z$. If $y<z$, one has $\sum_{y \leq \eta<z} \psi(\eta) \delta y \simeq 0$, so $\psi(z)=\psi(y)+\sum_{y \leq \eta<z} \psi^{[1]}(\eta) \delta y \simeq \psi(y)$. If $z<y_{0}$, the proof that $\psi(z) \simeq \psi(y)$ is analogous. Hence $\psi$ is $S$-continuous. Combining, we see that $\psi$ is of class $S^{0}$. We conclude that $\psi$ is of class $S^{n+1}$.

As a consquence we obtain a sort of minimal condition for functions to be of class $S^{n}$.

Theorem 3.7 Let $n \in \mathbb{N}$ be standard and $\psi: \mathbb{Y} \rightarrow \mathbb{R}$. Assume $\psi^{[n]}$ is of class $S^{0}$ and for some limited $y_{0} \in \mathbb{Y}$ it holds that $\psi\left(y_{0}\right), \psi^{[1]}\left(y_{0}\right), \cdots, \psi^{[n-1]}\left(y_{0}\right)$ are all limited. Then $\psi$ is of class $S^{n}$.

Proof By external induction. The case $n=0$ is trivial. Let $n$ be standard and assume the theorem is proved for $n$. Let $\psi$ be such that $\psi^{[n+1]}$ is of class $S^{0}$ and for some limited $y_{0} \in \mathbb{Y}$ it holds that $\psi\left(y_{0}\right), \psi^{[1]}\left(y_{0}\right), \cdots, \psi^{[n+1]}\left(y_{0}\right)$ are all limited. One observes that $\psi^{[1]}\left(y_{0}\right), \psi^{[2]}\left(y_{0}\right), \cdots, \psi^{[n+1]}\left(y_{0}\right)$ are all limited, and that $\psi^{[1][n]}=\psi^{[n+1]}$ is of class $S^{0}$. By the induction hypothesis $\psi^{[1]}$ is of class $S^{n}$. Then $\psi$ is of class $S^{n+1}$ by the Lemma of the discrete integral.

We now consider the stability of the class $S^{n}$ under algebraic operations. The proof of the first proposition is immediate. 
Proposition 3.8 Let $n \in \mathbb{N}$ be standard and $f, g: \mathbb{Y} \rightarrow \mathbb{R}$ be functions of class $S^{n}$. Let $a \in \mathbb{R}$ be limited. Then $f+g$ and af are of class $S^{n}$.

We formulate the product rule and the division rule first for functions defined on an equidistant near-continuum $\mathbb{X}$. Then we discuss its validity on general near-continua. Note that

$$
\begin{aligned}
\frac{\delta(f \cdot g)}{\delta \xi}(\xi) & =\frac{\delta f}{\delta \xi}(\xi) \cdot g(\xi+\delta \xi)+f(\xi) \cdot \frac{\delta g}{\delta \xi}(\xi) \\
& =\frac{\delta f}{\delta \xi}(\xi) \cdot g(\xi)+\frac{\delta f}{\delta \xi}(\xi) \cdot \frac{\delta g}{\delta \xi}(\xi) \delta \xi+f(\xi) \cdot \frac{\delta g}{\delta \xi}(\xi)
\end{aligned}
$$

Proposition 3.9 Let $n \in \mathbb{N}$ be standard and $f, g: \mathbb{X} \rightarrow \mathbb{R}$ be functions of class $S^{n}$. Then $f \cdot g$ is of class $S^{n}$.

Proof By external induction. The case $n=0$ follows from Proposition 2.6. Assume the property is valid for some standard integer $n$. Suppose $f$ and $g$ are of class $S^{n+1}$. The functions $f$ and $g$ are of class $S^{n}$ by Proposition 3.3. By the Lemma of the discrete derivative $\frac{\delta f}{\delta \xi}$ and $\frac{\delta g}{\delta \xi}$ are of class $S^{n}$. Then $\frac{\delta f}{\delta \xi} \cdot g, \frac{\delta f}{\delta \xi} \cdot \frac{\delta g}{\delta \xi}$ and $f \cdot \frac{\delta g}{\delta \xi}$ are of class $S^{n}$ by the induction hypothesis. By (1) and Proposition 3.8 the difference quotient $\frac{\delta(f \cdot g)}{\delta \xi}$ is of class $S^{n}$. Because $f \cdot g$ is of class $S^{0}$ we deduce from the Lemma of the discrete integral that $f \cdot g$ is of class $S^{n+1}$.

Lemma 3.10 Let $n \in \mathbb{N}$ be standard and $f: \mathbb{X} \rightarrow \mathbb{R}$ be a function of class $S^{n}$ with appreciable values for limited arguments. Let $A \subseteq \mathbb{X}$ be such that $A^{(n)} \neq \varnothing$ and $f \not 00$ on the limited part of $A$. Then $\frac{1}{f}$ is of class $S^{n}$ on $A$.

Proof By external induction. The case $n=0$ follows from Proposition 2.6. Assume the property is valid for some standard integer $n$. Let $f$ be a function of class $S^{n+1}$ and $A \subseteq \mathbb{X}$ be such that $A^{(n+1)} \neq \varnothing$ and $f$ takes appreciable values for limited arguments $\xi \in A$. Now

$$
\frac{\delta(1 / f)}{\delta \xi}(\xi)=-f^{[1]}(\xi) \cdot \frac{1}{f^{2}(\xi)-f(\xi) f^{[1]}(\xi) \delta \xi} .
$$

By Proposition 3.3 the function $f$ is of class $S^{n}$ on $A$ and by the Lemma of the discrete derivative $f^{[1]}$ is of class $S^{n}$ on $A^{(1)}$. Then $f^{2}(\xi)-f(\xi) f^{[1]}(\xi) \delta \xi$ is of class $S^{n}$ on $A^{(1)}$ by Proposition 3.9. Then $1 /\left(f^{2}(\xi)-f(\xi) f^{[1]}(\xi) \delta \xi\right)$ is of class $S^{n}$ on $A^{(1)}$ by the 
induction hypothesis. Hence $\frac{\delta(1 / f)}{\delta \xi}$ is of class $S^{n}$ on $A^{(1)}$. Because $\frac{1}{f}$ is of class $S^{0}$ on $A$, the function $\frac{1}{f}$ is of class $S^{n+1}$ on $A$ as a consequence of the Lemma of the discrete integral.

Proposition 3.11 Let $n \in \mathbb{N}$ be standard and $f, g: \mathbb{X} \rightarrow \mathbb{R}$ be functions of class $S^{n}$. Let $A \subseteq \mathbb{X}$ be such that $A^{(n)} \neq \varnothing$ and $g \not 0$ on the limited part of $A$.Then $f / g$ is of class $S^{n}$ on $A$.

The following example shows somewhat surprisingly that a very elementar function does not need to be of class $S^{2}$ on too irregular grids.

Example 3.12 There exists a strictly increasing function $\phi$ of class $S^{0}$ such that, with $\mathbb{Y} \equiv \phi(\mathbb{X})$, the quadratic function $\psi: \mathbb{Y} \rightarrow R$ defined by $\psi(\eta)=\eta^{2}$ is not of class $S^{2}$ on $\mathbb{Y}$. To see this, we will only consider the restriction of such a function $\phi$ to $\{0, \delta \xi, 2 \delta \xi\}$. Assume $\phi$ satisfies $\phi(0)=1, \phi(\delta \xi)=1+\delta \xi$ and $\phi(2 \delta \xi)=1+\delta \xi+\delta \xi^{\prime}$, where $\delta \xi^{\prime}>0$ is infinitesimal. The function $\psi$ is clearly of class $S^{0}$ on $Y \equiv\left\{1,1+\delta \xi, 1+\delta \xi+\delta \xi^{\prime}\right\}$, and also of class $S^{1}$, since

$$
\psi^{[1]}(1+\delta \xi)=\frac{\psi\left(1+\delta \xi+\delta \xi^{\prime}\right)-\psi(1+\delta \xi)}{\delta \xi^{\prime}}=2+2 \delta \xi+\delta \xi^{\prime},
$$

which is limited and nearly equal to

$$
\psi^{[1]}(1)=\frac{\psi(1+\delta \xi)-\psi(1)}{\delta \xi}=2+\delta \xi
$$

But whether $\psi$ is of class $S^{2}$ depends on the relation between $\delta \xi$ and $\delta \xi^{\prime}$. Indeed,

$$
\psi^{[2]}(1)=\frac{\psi^{[1]}(1+\delta \xi)-\psi^{[1]}(1)}{\delta \xi}=\frac{\delta \xi+\delta \xi^{\prime}}{\delta \xi}=1+\frac{\delta \xi^{\prime}}{\delta \xi} .
$$

One should wish that $\psi^{[2]}(1) \simeq 2$, but this is only realised if the grid $Y$ has some regularity, in particular if $\delta \xi^{\prime} / \delta \xi \simeq 1$. Clearly $\psi$ is not of class $S^{2}$ if $\psi^{[2]}(1)$ is unlimited; this happens when $\delta \xi^{\prime} / \delta \xi \simeq+\infty$, say for $\delta \xi^{\prime}=\sqrt{\delta \xi}$.

Example 3.12 only apparently contradicts the Lemma of the discrete integral, since $\psi^{[1]}(\phi(\xi))=2 \phi(\xi)+\delta \phi(\xi)$. This shows that $\psi$ is a discrete primitive of $\eta \longmapsto 2 \eta+\delta \eta$, which is not of class $S^{1}$ on $\mathbb{Y}$ if $\delta \eta / \delta \xi \simeq+\infty$, and not a discrete primitive of $\eta \longmapsto 2 \eta$, which is of class $S^{n}$ for all standard $n$.

Example 3.12 shows also that the product rule does not hold in all generality: if $f$ and $g$ are of class $S^{n}$ on some set $\mathbb{Y}$ for some limited $n$, the product $f \cdot g$ does not need to 
be of class $S^{n}$. It is easy to verify that the division rule also does not hold in general. Indeed, with $f(x)=1, g(x)=x, \chi(x) \equiv f(x) / g(x)=1 / x$ and $\phi$ as above one finds

$$
\chi^{[2]}(1)=\frac{1+\delta \xi^{\prime} / \delta \xi}{(1+\delta \xi)\left(1+\delta \xi+\delta \xi^{\prime}\right)}
$$

Hence $\chi^{[2]}(1)$ is unlimited again for $\delta \xi^{\prime}=\sqrt{\delta \xi}$. We will show in Subsection 5.2 that the product rule and the division rule do hold provided $\mathbb{Y}$ is the image of $\mathbb{X}$ by some function $\phi$ which is of class $S^{n}$ itself.

On the other hand it follows from the Propositions 3.8 and 3.9 that polynomials of standard degree with limited coefficients defined on the equidistant continuum $\mathbb{X}$ are of class $S^{n}$ for all standard $n$. Then by Proposition 3.11 a rational function which is a quotient of polynomials of standard degree and limited coefficients is of class $S^{n}$ on all sets of the form $A^{(n)} \neq \varnothing$ such that $A \subseteq \mathbb{X}$ and no element of $A$ is infinitely close to a limited singularity of the denominator.

We verify that the two examples of discrete functions $\mathcal{E}$ and $\mathcal{F}$ defined in Section 2 are of class $S^{n}$ for all standard $n$.

Indeed, because

$$
\frac{\delta \mathcal{E}(\xi)}{\delta \xi}=\mathcal{E}(\xi)
$$

and $\mathcal{E}$ is of class $S^{0}$, its discrete derivative $\frac{\delta \mathcal{E}}{\delta \xi}$ is also of class $S^{0}$. This implies that $\mathcal{E}$ is of class $S^{1}$. Then $\frac{\delta \mathcal{E}}{\delta \xi}$ is also class $S^{1}$, so by the Lemma of the discrete integral $\mathcal{E}$ is of class $S^{2}$. With external induction, applying this procedure to the induction step, one shows that the function $\mathcal{E}$ is of class $S^{n}$ for all standard $n \in \mathbb{N}$.

Secondly, for $\xi \in \mathbb{X}^{+}$

$$
\frac{\delta \mathcal{F}(\xi)}{\delta \xi}=\xi \cdot \mathcal{F}(\xi)
$$

We already saw that $\mathcal{F}$ is of class $S^{0}$ on $\mathbb{X}^{+}$. Because the monomial $\xi$ is of class $S^{n}$ for all standard $n$ and these classes are stable under multiplication, we may apply the same method as above to show that $\mathcal{F}$ is of class $S^{n}$ on $\mathbb{X}^{+}$, for all standard $n$.

\section{Transition from the discrete to the continuous in one vari- able on equidistant near-continua}

Let $\mathbb{X}$ be an equidistant near-continuum as above. We consider first discrete functions of class $S^{n}$ on the whole of $\mathbb{X}$ and extend the Theorem of the continuous shadow to transitions from the discrete to the continuous of higher order of regularity. 
The case for differentiability of first order had already been proved earlier [8] [4].

Theorem 4.1 (Theorem of the differentiable shadow) Let $\phi: \mathbb{X} \rightarrow \mathbb{R}$ be of class $S^{1}$. Then its shadow $f$ is a real function of class $C^{1}$ and $\phi^{[1]}(\xi) \simeq f^{\prime}(\xi)$ for all limited $\xi \in \mathbb{X}$.

N.B. The definition of function of class $S^{1}$ in [8] is different of ours. The essential difference is that it concerns only functions defined on $\mathbb{R}$. However, this difference is not very important since a discrete function defined on $\mathbb{X}$ can always be appropriately extended to a real function $\bar{f}$ of class $S^{1}$ defined on the whole of $\mathbb{R}$.

The proof that the shadow of a function of class $S^{n}$ defined on $\mathbb{X}$ is a function of class $C^{n}$ is contained in [11] and [6] and will be repeated here.

Theorem 4.2 Let $n \in \mathbb{N}$ be standard and let $\phi: \mathbb{X} \rightarrow \mathbb{R}$ be of class $S^{n}$. Then its shadow is a function $f: \mathbb{R} \rightarrow \mathbb{R}$ of class $C^{n}$ and $\phi^{[n]}(\xi) \simeq f^{(n)}(\xi)$ for all limited $\xi \in \mathbb{X}$.

Proof By external induction. If $n=0$, by the Theorem of the continuous shadow $f$ is a real function of class $C^{0}$ and $\phi(\xi) \simeq f(\xi)$ for all limited $\xi \in \mathbb{X}$. Assume the property is valid for some standard integer $n$. Let $\phi$ be a function of class $S^{n+1}$. By Proposition 3.5 the function $\phi^{[n]}$ is of class $S^{1}$. By the induction hypothesis its shadow equals $f^{(n)}$. By the Theorem of the differentiable shadow $f^{(n)}$ is continuously differentiable and $\left(\phi^{[n]}\right)^{[1]}(\xi) \simeq\left(f^{(n)}\right)^{\prime}(\xi)$ for all limited $\xi \in \mathbb{X}$. Hence $\phi^{[n+1]}(\xi) \simeq f^{(n+1)}(\xi)$ for all limited $\xi \in \mathbb{X}$.

As a consequence we obtain that for pairs of functions of class $S^{n}$ the property of near-equality is hereditary to discrete derivatives up to order $n$.

Theorem 4.3 Let $n \in \mathbb{N}$ be standard and $f, g: \mathbb{X} \rightarrow \mathbb{R}$ be two functions of class $S^{n}$ such that $f(\xi) \simeq g(\xi)$ for all limited $\xi \in \mathbb{X}$. Then $f^{[n]}(\xi) \simeq g^{[n]}(\xi)$ for all limited $\xi \in \mathbb{X}$.

Proof By the Theorem of the continuous shadow ${ }^{\circ} f={ }^{\circ} g$. If $n>0$, by Theorem 4.2

$$
f^{[n]}(\xi) \simeq{ }^{\circ} f^{(n)}(\xi)={ }^{\circ} g^{(n)}(\xi) \simeq g^{[n]}(\xi) .
$$




\section{Transition from the discrete to the continuous on general near-continua}

We consider now functions of class $S^{n}$ on possibly non-equidistant near-continua. The next theorem gives conditions for near-equality of such functions and their difference quotients to their shadows and the respective derivatives of these shadows.

Theorem 5.1 Let $n \in \mathbb{N}$ be standard. Let $\bar{\xi} \in \mathbb{X}$ be limited. Let $\phi: \mathbb{X} \rightarrow \mathbb{R}$ be a function of class $S^{n}$ such that $\phi^{[1]}(\bar{\xi}) \neq 0$. Let $\psi: \phi(\mathbb{X}) \rightarrow \mathbb{R}$ be a function of class $S^{n}$. Let $\chi=\psi \circ \phi$. Then there exists a near-interval $[\alpha \cdot \beta]$ with $\alpha, \beta \in \mathbb{X}$ limited and $\alpha \succsim \bar{\xi} \succsim \beta$ such that $\phi^{[1]}(\xi) \neq 0$ for all $\xi \in[\alpha \cdot \beta]$, and the function $\chi$ is of class $S^{n}$ on $[\alpha \cdots \beta]$, with for all $\xi \in[\alpha \cdots \beta]$

$$
\chi^{[n]}(\xi) \simeq \sum_{k_{1}+2 k_{2}+\ldots+n k_{n}=n} \frac{n !}{k_{1} ! k_{2} ! \ldots k_{n} !} \psi^{\left[k_{1}+k_{2}+\ldots+k_{n}\right]}(\phi(\xi)) \prod_{i=1}^{n}\left(\frac{\phi^{[i]}(\xi)}{i !}\right)^{k_{i}} .
$$

Moreover, let $f={ }^{\circ} \phi, g={ }^{\circ} \psi$ and $h={ }^{\circ} \chi$. Let $a={ }^{\circ} \alpha$ and $b={ }^{\circ} \beta$. Then $g$ is of class $C^{n}$ on $[f(a), f(b)]$ with $g^{(n)}={ }^{\circ}\left(\psi^{[n]}\right)$ and $h$ is of class $C^{n}$ on $[a, b]$, with for all $x \in[a, b]$

$$
h^{(n)}(x)=\sum_{k_{1}+2 k_{2}+\ldots+n k_{n}=n} \frac{n !}{k_{1} ! k_{2} ! \ldots k_{n} !} g^{\left(k_{1}+k_{2}+\ldots+k_{n}\right)}(f(x)) \prod_{i=1}^{n}\left(\frac{f^{(i)}(x)}{i !}\right)^{k_{i}} .
$$

Formula (3) is known as the formula of Faà di Bruno. It extends the Chain Rule to derivatives of higher order. Thus formula (2) is a discrete, approximative version of the formula of Faà di Bruno.

One of the consequences of the theorem above is that a sufficient condition for a function $\psi$ to be of class $S^{n}$ on some near-continuum $\mathbb{Y} \subset \mathbb{R}$, with the $n^{\text {th }}$-order difference quotient infinitely close to the $n^{\text {th }}$-order derivative of its shadow, is that $\mathbb{Y}$ is the image of an equidistant near-continuum $\mathbb{X} \subset \mathbb{R}$ by a (locally) strictly monotone function $\phi$ which is itself of class $S^{n}$. We already saw that absence of such a regularity condition may even lead to difference quotients with infinitely large values, which certainly are not nearly-equal to derivatives of ${ }^{\circ} \psi$. A near-continuum $\mathbb{Y} \subset \mathbb{R}$ which is the image of an equidistant near-continuum $\mathbb{X} \subset \mathbb{R}$ by a monotone function $\phi$ of class $S^{n}$ will be called a near-continuum of class $S^{n}$.

Below we prove formula (2) along the lines of the proof of De la Vallée-Poussin (see also [12]) of the usual formula of Faà di Bruno (3). 
The proof of De la Vallée-Poussin is by induction. The first step consists in proving that the coefficients of $g^{\left(k_{1}+k_{2}+\ldots+k_{n}\right)}(f(x)) \prod_{i=1}^{n}\left(\frac{f^{(i)}(x)}{i !}\right)^{k_{i}}$ are integers, and independent of $f$ and $g$. This means that the coefficients may be determined for convenient special functions, in fact powers of polynomials of the form $\left(a_{1} x+a_{2} x^{2}+\ldots+a_{n} x^{n}\right)^{k}$. This step uses the multinomial expansion

$$
\left(x_{1}+x_{2}+\ldots+x_{n}\right)^{k}=\sum_{k_{1}+k_{2}+\ldots+k_{n}=k} \frac{k !}{k_{1} ! k_{2} ! \ldots k_{n} !} x_{1}^{k_{1}} x_{2}^{k_{2}} \ldots x_{n}^{k_{n}} .
$$

Finally the powers of polynomials are repeatedly differentiated.

In the discrete case some complications arise from the fact that difference quotients are not always taken at $\xi$, but also at points $\xi+\theta \delta \xi$, with $\theta>0$. Hence the product of powers at the end of the formula of Faà di Bruno transforms into a product of products; but we will show that the latter products are infinitely close to the corresponding powers taken at $\xi$.

\subsection{Properties of the composition function}

Convention, notations. Let $n \in \mathbb{N}$ be standard. Let $\bar{\xi} \in \mathbb{X}$ be limited. Let $\phi: \mathbb{X} \rightarrow \mathbb{R}$ be a function of class $S^{n}$ such that $\phi^{[1]}(\bar{\xi}) \not 0$. As a consequence of the principle of Fehrele [8] there exists a near-interval $[\alpha \cdots \beta]$ with $\alpha, \beta \in \mathbb{X}$ limited and $\alpha \succsim \bar{\xi} \succsim \beta$ such that $\phi^{[1]}(\xi) \not \chi 0$ for all $\xi \in[\alpha \cdots \beta]$. Let $1 \leq m \leq n$. Without restriction of generality we assume that $\phi^{[1]}(\xi) \gtrsim 0$ for all $\xi \in[\alpha \cdot \beta]$ and that $\phi^{[m]}(\xi)$ is defined on $[\alpha \cdot \beta]$ instead of $[\alpha \cdot \beta]^{(m)}$. Notice that $\phi(\alpha) \succsim \phi(\bar{\xi}) \succsim \phi(\beta)$.

We use the following notations. Let $\psi: \phi(\mathbb{X}) \rightarrow \mathbb{R}$ be a function of class $S^{n}$ and let $\chi=\psi \circ \phi$. All functions being of class $S^{0}$, their shadows are well-defined and we put $f={ }^{\circ} \phi, g={ }^{\circ} \psi$ and $h={ }^{\circ} \chi$. Let $a={ }^{\circ} \alpha$ and $b={ }^{\circ} \beta$, and $c \equiv f(a)={ }^{\circ}(\phi(\alpha))$ and $d \equiv f(b)={ }^{\circ}(\phi(\beta))$. Then $f$ is of class $C^{n}$ on $[a, b]$, the function $g$ is at least of class $C^{0}$ on $[f(a), f(b)]$ and $h=f \circ g$ is at least of class $C^{0}$ on $[a, b]$.

The discrete Chain Rule for the first-order difference quotient of the composite function $\chi$ takes the form

$$
\chi^{[1]}(\xi)=\psi^{[1]}(\phi(\xi)) \phi^{[1]}(\xi) .
$$

This formula is used in the proof that the composition of two functions of class $S^{n}$ is also of class $S^{n}$.

Proposition 5.2 Let $n \in \mathbb{N}$ be standard. Then $\chi$ is of class $S^{n}$ on $[\alpha \cdots \beta]$ and $h$ is of class $C^{n}$ on $[a, b]$. 
Proof With external induction. The composition of two functions of class $S^{0}$ is clearly of class $S^{0}$. Assume the proposition is proved for $n-1$. Let $\chi=\psi \circ \phi$, with $\psi$ and $\phi$ of class $S^{n}$, then in particular $\chi$ is of class $S^{0}$ on $[\alpha \cdots \beta]$. Also $\phi$ of class $S^{n-1}$. By the Lemma of the discrete derivative $\phi^{[1]}$ is of class $S^{n-1}$ on $[\alpha \cdots \beta]$ and $\psi^{[1]}$ is of class $S^{n-1}$ on $[\phi(\alpha) \cdots \phi(\beta)]$. By the Induction Hypothesis $\psi^{[1]} \circ \phi$ is of class $S^{n-1}$ on $[\alpha \cdots \beta]$ and then $\chi^{[1]}$ is of class $S^{n-1}$ on $[\alpha \cdots \beta]$ by (4) and by Proposition 3.9. Then it follows from the Lemma of the discrete integral that $\chi$ is of class $S^{n}$ on $[\alpha \cdots \beta]$. Then Theorem 4.2 implies that $h$ is of class $C^{n}$ on $[a, b]$.

We already saw that not on all near-continua $\phi(\mathbb{X})$ the class $S^{n}$ is stable under the usual algebraic operations. We show that this stability does hold for functions defined on near-continua of class $S^{n}$.

We continue to assume the conventions which where introduced above and prove first a lemma.

Lemma 5.3 Let $n \in \mathbb{N}, n \geq 1$ be standard, $\mathbb{Y} \equiv \eta(\mathbb{X})$ be a near-continuum of class $S^{n}$ and $f: \mathbb{Y} \rightarrow \mathbb{R}$ be a function of class $S^{n}$. Define $g: \mathbb{Y} \rightarrow \mathbb{R}$ by $g(\eta)=f(\eta+\delta \eta)$. Then $g$ is of class $S^{n-1}$ in $\eta$.

Proof The case $n=1$ follows from the fact that $\delta \eta_{\xi}$ is infinitesimal: the number $\eta_{\xi}+\delta \eta_{\xi}$ is limited whenever $\eta_{\xi}$ is limited. Assume that $n>1$. By the Lemma of the discrete derivative $f^{[1]}$ is of class $S^{n-1}$ in $\eta$ and $\eta^{[1]}$ is of class $S^{n-1}$ in $\xi$. Also

$$
g\left(\eta_{\xi}\right)=f\left(\eta_{\xi}+\delta \eta_{\xi}\right)=f^{[1]}\left(\eta_{\xi}\right) \eta^{[1]}(\xi) \delta \xi+f\left(\eta_{\xi}\right) .
$$

Then by Proposition 5.2 and Proposition 3.9 the function $\chi$ defined by $\chi(\xi)=$ $f^{[1]}\left(\eta_{\xi}\right) \eta^{[1]}(\xi)$ is of class $S^{n-1}$ in $\xi$. Let $k$ be such that $1 \leq k \leq n-1$. Then $\chi^{[k]}(\xi) \delta \xi$ is infinitesimal and

$$
g^{[k]}\left(\eta_{\xi}\right)=\chi^{[k]}(\xi) \delta \xi+f^{[k]}\left(\eta_{\xi}\right) \simeq f^{[k]}\left(\eta_{\xi}\right),
$$

which is of class $S^{0}$ in $\eta$. Then $g^{[k]}$ is of class $S^{0}$ in $\eta$ by Proposition 2.3. Hence $g$ is of class $S^{n}$ in $\eta$.

Proposition 5.4 Let $n \in \mathbb{N}$ be standard, $\mathbb{Y}$ be a near-continuum of class $S^{n}$ and $f, g: \mathbb{Y} \rightarrow \mathbb{R}$ be functions of class $S^{n}$. Then $f \cdot g$ is of class $S^{n}$.

Proof By external induction. The case $n=0$ is contained in Proposition 2.6. Assume the property is valid for some standard integer $n$. Suppose $f, g$ and $\eta$ are of class $S^{n+1}$. 
By Lemma 5.3 the function $g(\eta+\delta \eta)$ is of class $S^{n}$. By the Lemma of the discrete derivative $f^{[1]}$ and $g^{[1]}$ are of class $S^{n}$ and $\eta^{[1]}$ is of class $S^{n}$. Now

$$
(f \cdot g)^{[1]}(\eta)=f^{[1]}(\eta) g(\eta+\delta \eta)+f(\eta) g^{[1]}(\eta) .
$$

Hence $(f \cdot g)^{[1]}$ is of class $S^{n}$. Because $f \cdot g$ is of class $S^{0}$, the Lemma of discrete integration implies that the function $f \cdot g$ is of class $S^{n+1}$.

Next lemma is proved similarly, now using the formula

$$
\frac{\delta(1 / f)}{\delta \eta}(\eta)=-f^{[1]}(\eta) \cdot \frac{1}{f(\eta) f(\eta+\delta \eta)}
$$

Lemma 5.5 Let $n \in \mathbb{N}$ be standard, $\mathbb{Y}$ be a near-continuum of class $S^{n}$ and $f: \mathbb{Y} \rightarrow \mathbb{R}$ be a function of class $S^{n}$. Let $A \subseteq \mathbb{Y}$ be such that $A^{(n)} \neq \varnothing$ and $f \not z 0$ on the limited part of $A$.Then $\frac{1}{f}$ is of class $S^{n}$ on $A$.

Proposition 5.6 Let $n \in \mathbb{N}$ be standard, $\mathbb{Y}$ be a near-continuum of class $S^{n}$ and $f, g: \mathbb{Y} \rightarrow \mathbb{R}$ be functions of class $S^{n}$. Let $A \subseteq \mathbb{Y}$ be such that $A^{(n)} \neq \varnothing$ and $g \not 0$ on the limited part of $A$.Then $f / g$ is of class $S^{n}$ on $A$.

Proof By Proposition 5.4 and Lemma 5.5.

The proposition implies that, for all standard $n$, all polynomials of standard degree with limited coefficients defined on some near-continuum $\mathbb{Y}$ of class $S^{n}$ are of class $S^{n}$, and all rational functions which are quotients of such polynomials are of class $S^{n}$ on all sets of the form $A^{(n)} \neq \varnothing$, such that the elements of $A \subseteq \mathbb{Y}$ are not infinitely close to a limited singularity of the denominator.

As already said such functions do not need to be of class $S^{n}$ for all standard $n \in \mathbb{N}$ on too irregular near-continua $\phi(\mathbb{X})$. Next theorem says that a sufficiently sparse subset $\mathbb{S}$ of a near-continuum $\phi(\mathbb{X})$ of class $S^{0}$ may be determined which happens to be a near-continuum of class $S^{n}$ for all standard $n \in \mathbb{N}$. Then the restrictions of the functions mentioned beforehand to $\mathbb{S}$ will be of class $S^{n}$ for all standard $n \in \mathbb{N}$ indeed.

Theorem 5.7 Let $\phi(\mathbb{X})$ be a near-continuum of class $S^{0}$. Then there exists $\delta y>$ $0, \delta y \simeq 0$, which is a multiple of $\delta \xi$, such that $\psi(\mathbb{Y}) \subseteq \phi(\mathbb{X})$ is a near continuum of class $S^{n}$ for all standard $n \in \mathbb{N}$, where $\mathbb{Y} \equiv \mathbb{Z} \delta y$ and $\psi: \mathbb{Y} \rightarrow \phi(\mathbb{X})$ is defined by

$$
\psi(y)=\min \{\eta \in \phi(\mathbb{X}) \mid \eta \geq y\} .
$$


Proof Applying the Cauchy Principle twice there is $\epsilon>0, \epsilon \simeq 0$ such that $\delta \phi(\xi)<\epsilon$ for all limited $\xi \in \mathbb{X}$. By Robinson's Lemma there is $\omega \simeq+\infty$ such $\delta y \equiv \epsilon^{1 / \omega} \simeq 0$; we may suppose that $\delta y$ is a multiple of $\delta \xi$. Put $\mathbb{Y}=\mathbb{Z} \delta y$. Observe that if $y \in \mathbb{Y}$ is limited

$$
\psi(y)-y \leq \epsilon=\delta y^{\omega} .
$$

Hence $\psi(y)-y$ is an element of the external set of all infinitely large powers of $\delta y$, which we denote by $£ \delta y^{\bar{\infty}}$; this external set is stable by divisions by standard powers of $\delta y$.

We prove with external induction that $\psi^{[n]}(y)-I^{[n]}(y) \in £ \delta y^{\bar{\infty}}$ for all limited $y \in \mathbb{Y}$ and for all standard $n \in \mathbb{N}$, where $I$ is the identity function on $\mathbb{Y}$. For $n=0$ the property follows from (5). For $n=1$ one has, for some $\epsilon_{1}$ with $0 \leq \epsilon_{1} \leq \epsilon$

$$
\psi^{[1]}(y)-y^{[1]}=\frac{y+\delta y+\epsilon_{1}-y}{\delta y}-1 \in £ \delta y^{\bar{\infty}} .
$$

For $n=2$ one has $y^{[2]}=0$ and $\psi^{[1]}(y), \psi^{[1]}(y+\delta y) \in 1+£ \delta y^{\bar{\infty}}$. Hence

$$
\psi^{[2]}(y)-y^{[2]}=\psi^{[2]}(y) \in \frac{1+£ \delta y^{\bar{\infty}}-\left(1+£ \delta y^{\bar{\infty}}\right)}{\delta y} \subseteq £ \delta y^{\bar{\infty}} .
$$

Then for $n=3$

$$
\psi^{[3]}(y)-y^{[3]}=\psi^{[3]}(y) \in \frac{£ \delta y^{\bar{\infty}}-£ \delta y^{\bar{\infty}}}{\delta y} \subseteq £ \delta y^{\bar{\infty}},
$$

a property which will also hold for the remaining standard natural numbers. We conclude that $\psi$ and $\psi^{[n]}$ are of class $S^{0}$ for all standard $n \in \mathbb{N}$. As a consequence $\psi$ is of class $S^{n}$ for all standard $n \in \mathbb{N}$ and $\psi(\mathbb{Y})$ is a near continuum of class $S^{n}$ for all standard $n \in \mathbb{N}$.

We end this section by showing that formula (2) holds for the $n^{\text {th }}$ order difference quotient of the composite function $\chi$.

Lemma 5.8 Let $n \in \mathbb{N}$ be standard and $\xi \in[\alpha \cdot \beta]$. Then $\chi^{[n]}(\xi)$ is the sum of a standard finite number of terms consisting of products with a standard finite number of factors of the form

$$
\psi^{[k]}(\phi(\xi)) \prod_{j=1}^{k_{1}} \phi^{[1]}\left(\xi+\theta_{1 j} \delta \xi\right) \prod_{j=1}^{k_{2}} \phi^{[2]}\left(\xi+\theta_{2 j} \delta \xi\right) \ldots \prod_{j=1}^{k_{n}} \phi^{[n]}\left(\xi+\theta_{n j} \delta \xi\right),
$$

where $k_{1}+k_{2}+\ldots+k_{n} \equiv k$ and $k_{1}+2 k_{2}+\ldots+n k_{n}=n$, and $0 \leq \theta_{i j} \leq n$ for all $i, j$ with $1 \leq j \leq k_{i}$ and $1 \leq i \leq n$. 
Proof By external induction. Firstly, let $n=1$. Then $k=k_{1}=1 \cdot k_{1}=n=1$ and

$$
\chi^{[1]}(\xi)=\psi^{[1]}(\phi(\xi)) \phi^{[1]}(\xi)=\psi^{[k]}(\phi(\xi)) \phi^{\left[k_{1}\right]}\left(\xi+\theta_{11} \delta \xi\right),
$$

with $\theta_{11}=0 \leq n$.

Assume the property has been proved up to some standard $n$. Applying the product rule (1), starting with the discrete derivative of $\psi^{[k]}(\phi(\xi))$, the first term, say $\tau_{k+1}(\xi)$, becomes

$$
\begin{aligned}
\tau_{k+1}(\xi)= & \psi^{[k+1]}(\phi(\xi))\left(\phi^{[1]}(\xi) \prod_{j=1}^{k_{1}} \phi^{[1]}\left(\xi+\left(\theta_{1 j}+1\right) \delta \xi\right)\right) \times \\
& \prod_{j=1}^{k_{2}} \phi^{[2]}\left(\xi+\left(\theta_{2 j}+1\right) \delta \xi\right) \ldots \prod_{j=1}^{k_{n}} \phi^{[n]}\left(\xi+\left(\theta_{n j}+1\right) \delta \xi\right) .
\end{aligned}
$$

We define $k_{1}^{\prime}=k_{1}+1, k_{2}^{\prime}=k_{2}, \ldots, k_{n}^{\prime}=k_{n}$ and $k_{n+1}^{\prime}=0$. We let $k^{\prime}=k+1$ and we define $\theta_{11}^{\prime}=0, \theta_{1 j}^{\prime}=\theta_{1 j-1}+1$ for all $j$ with $2 \leq j \leq k_{1}, \theta_{i j}^{\prime}=\theta_{i j}+1$ for all $i, j$ with $1 \leq j \leq k_{i}$ and $2 \leq i \leq n$ and $\theta_{n+1 j}^{\prime}=0$. Then $k_{1}^{\prime}+k_{2}^{\prime}+\ldots+k_{n}^{\prime} \equiv k^{\prime}$ and $k_{1}^{\prime}+2 k_{2}^{\prime}+\ldots+(n+1) k_{n+1}^{\prime}=n+1$, and $0 \leq \theta_{i j}^{\prime} \leq n+1$ for all $i, j$ with $1 \leq j \leq k_{i}^{\prime}$ and $1 \leq i \leq n+1$. Further

$$
\begin{aligned}
\tau_{k+1}(\xi)= & \psi^{\left[k^{\prime}\right]}(\phi(\xi)) \prod_{j=1}^{k_{1}^{\prime}} \phi^{[1]}\left(\xi+\theta_{1 j}^{\prime} \delta \xi\right) \prod_{j=1}^{k_{2}^{\prime}} \phi^{[2]}\left(\xi+\theta_{2 j}^{\prime} \delta \xi\right) \times \\
& \ldots \times \prod_{j=1}^{k_{n+1}^{\prime}} \phi^{[n+1]}\left(\xi+\theta_{n+1 j}^{\prime} \delta \xi\right) .
\end{aligned}
$$

Continuing to apply the product rule, let $1 \leq m \leq k$, and suppose it is the turn of the $m^{\text {th }}$ factor of

$$
\Pi(\xi) \equiv \prod_{j=1}^{k_{1}} \phi^{[1]}\left(\xi+\theta_{1 j} \delta \xi\right) \prod_{j=1}^{k_{2}} \phi^{[2]}\left(\xi+\theta_{2 j} \delta \xi\right) \ldots \prod_{j=1}^{k_{n}} \phi^{[n]}\left(\xi+\theta_{n j} \delta \xi\right)
$$

to be differentiated discretely, which is, say, of the form $\phi^{[i]}\left(\xi+\theta_{i j} \delta \xi\right)$. Its discrete derivative is $\phi^{[i+1]}\left(\xi+\theta_{i j} \delta \xi\right)$. We define $k_{1}^{\prime}=k_{1}, \ldots, k_{i-1}^{\prime}=k_{i-1}, k_{i}^{\prime}=k_{i}-1, k_{i+1}^{\prime}=$ $k_{i+1}+1, k_{i+2}^{\prime}=k_{i+2}, \ldots, k_{n}^{\prime}=k_{n}$, and $k_{n+1}^{\prime}=0$ whenever $m \leq k-k_{n}$ and $k_{n+1}^{\prime}=1$ whenever $m>k-k_{n}$. We let $k^{\prime}=k$ and we define new $\theta_{i j}^{\prime}$ by adding nothing to the $\theta_{i j}$ occurring up to the $m^{\text {th }}$ factor, and adding 1 to to the $\theta_{i j}$ occurring after this factor, with $\theta_{n+1 j}^{\prime}=0$. The term corresponding to the discrete derivative of the $m^{\text {th }}$ factor becomes

$$
\psi^{\left[k^{\prime}\right]}(\phi(\xi)) \prod_{j=1}^{k_{1}^{\prime}} \phi^{[1]}\left(\xi+\theta_{1 j}^{\prime} \delta \xi\right) \prod_{j=1}^{k_{2}^{\prime}} \phi^{[2]}\left(\xi+\theta_{2 j}^{\prime} \delta \xi\right) \ldots \prod_{j=1}^{k_{n+1}^{\prime}} \phi^{[n+1]}\left(\xi+\theta_{n+1 j}^{\prime} \delta \xi\right),
$$


with $k_{1}^{\prime}+k_{2}^{\prime}+\ldots+k_{n}^{\prime} \equiv k^{\prime}$,

$$
\begin{aligned}
k_{1}^{\prime}+2 k_{2}^{\prime}+\ldots+i k_{i}^{\prime}+(i+1) k_{i+1}^{\prime}+(i+2) k_{i+2}^{\prime}+\ldots+(n+1) k_{n+1}^{\prime} & \\
=k_{1}+2 k_{2}+\ldots+i k_{i}-i+(i+1) k_{i+1}+i+1+ & (i+2) k_{i+1}+\ldots \\
& +(n+1) k_{n+1}=n+1,
\end{aligned}
$$

and $0 \leq \theta_{i j}^{\prime} \leq n+1$ for all $1 \leq j \leq k_{i}^{\prime}$ and $1 \leq i \leq n+1$. Every product has at most $n+1$ factors, and the number of terms in the induction step increases with at most $n+1$. In conclusion, the number of terms remains standard finite, each term having at most a standard number of factors.

Lemma 5.9 Let $n \in \mathbb{N}$ be standard. Then there are constants $C_{n, k_{1}, \ldots, k_{n}}$, which are standard integers independent from $\phi$ and $\psi$, such that $\chi^{[n]}(\xi)$ is of the form

$$
\chi^{[n]}(\xi) \simeq \sum_{k_{1}+2 k_{2}+\ldots+n k_{n}=n} C_{n, k_{1}, \ldots, k_{n}} \psi^{\left[k_{1}+k_{2}+\ldots+k_{n}\right]}(\phi(\xi)) \prod_{i=1}^{n}\left(\phi^{[i]}(\xi)\right)^{k_{i}},
$$

whenever $\xi \in[\alpha \cdot \beta]$.

Proof Lemma 5.8 states that $\chi^{[n]}(\xi)$ is a standard finite sum of standard finite products of the form

$$
\psi^{[k]}(\phi(\xi)) \prod_{j=1}^{k_{1}} \phi^{[1]}\left(\xi+\theta_{1 j} \delta \xi\right) \prod_{j=1}^{k_{2}} \phi^{[2]}\left(\xi+\theta_{2 j} \delta \xi\right) \ldots \prod_{j=1}^{k_{n}} \phi^{[n]}\left(\xi+\theta_{n j} \delta \xi\right),
$$

where $k_{1}+k_{2}+\ldots+k_{n}=k$ and $k_{1}+2 k_{2}+\ldots+n k_{n}=n$, and $0 \leq \theta_{i j} \leq n$ for all $i, j$ with $1 \leq j \leq k_{i}$ and $1 \leq i \leq n$; the form of the products does not depend on the individual properties of $\phi$ and $\psi$. Because all $\theta_{i j}$ satisfy $0 \leq \theta_{i j} \leq n$, all arguments $\xi+\theta_{i j} \delta \xi$ of the factors of the products above are nearly equal to $\xi$. Now $\psi^{[k]}(\phi(\xi))$, being a composition of two functions of class $S^{0}$, is limited, all functions $\phi^{[j]}$ with $1 \leq j \leq n$ are of class $S^{0}$ and there is only a standard finite number of factors in the products above. Hence each product satisfies

$$
\begin{aligned}
& \psi^{[k]}(\phi(\xi)) \prod_{j=1}^{k_{1}} \phi^{[1]}\left(\xi+\theta_{1 j} \delta \xi\right) \prod_{j=1}^{k_{2}} \phi^{[2]}\left(\xi+\theta_{2 j} \delta \xi\right) \ldots \prod_{j=1}^{k_{n}} \phi^{[n]}\left(\xi+\theta_{n j} \delta \xi\right) \\
\simeq & \psi^{[k]}\left(\phi(\xi) \prod_{i=1}^{n}\left(\phi^{[i]}(\xi)\right)^{k_{i}} .\right.
\end{aligned}
$$

This operation has the possible effect of regrouping terms with distinct products in some packet of identical powers, but the number of terms resulting from the regrouping does not depend on $\phi$ and $\psi$. Combining, the number of these terms in (8) is a function of $n$ and $k_{1}, \ldots, k_{n}$ only, which we may denote by $C_{n, k_{1}, \ldots, k_{n}}$. 
Observing that the coefficients $C_{n, k_{1}, \ldots, k_{n}}$ do not depend on $\phi$ and $\psi$, we choose convenient functions to determine them. As in the proof of the ordinary Faà di Bruno Theorem, we define for $k, n \in \mathbb{N}$ and $a_{1}, \cdots, a_{n} \in \mathbb{R}$

$$
\left\{\begin{array}{l}
p(\xi)=a_{1} \xi+\ldots+a_{n} \xi^{n} \\
m(\eta)=\eta^{k} \\
c(\xi)=(m \circ p)(\xi)=\left(a_{1} \xi+\ldots+a_{n} \xi^{n}\right)^{k}
\end{array}\right.
$$

It follows from the multinomial expansion that for all $\xi \in \mathbb{X}$

$$
c(\xi)=\sum_{k_{1}+k_{2}+\ldots+k_{n}=k} \frac{k !}{k_{1} ! k_{2} ! \ldots k_{n} !} \prod_{i=1}^{n} a_{i}^{k_{i}} \xi^{k_{1}+2 k_{2}+\ldots+n k_{n}} .
$$

We start with some lemmas.

Lemma 5.10 Let $k, n \in \mathbb{N}$ be standard with $k, n \geq 1$. Then

$$
\frac{\delta^{k} \xi^{n}}{\delta \xi^{k}}\left\{\begin{array}{lll}
\simeq 0 & & \xi=0, k<n \\
=n ! & & k=n \\
=0 & & k>n .
\end{array}\right.
$$

Proof By external induction in $n$. If $n=1$ the results are trivial. Assume the lemma has been proved for $n-1$. Observe that

$$
\frac{\delta \xi^{n}}{\delta \xi}=n \xi^{n-1}+\delta \xi q(\xi)
$$

where $q$ is a polynomial of degree $n-2$ with limited coefficients, containing powers of $\delta \xi$. Now

$$
\frac{\delta^{k} \xi^{n}}{\delta \xi^{k}}=n \frac{\delta^{k-1} \xi^{n-1}}{\delta \xi^{k-1}}+\delta \xi \frac{\delta^{k-1} q(\xi)}{\delta \xi^{k-1}} .
$$

As long as $k<n$ the induction hypothesis yields $\frac{\delta^{k-1} \xi^{n-1}}{\delta \xi^{k-1}} \simeq 0$ in $\xi=0$, and also $\delta \xi \frac{\delta^{k-1} q(\xi)}{\delta \xi^{k-1}} \simeq 0$ in $\xi=0$, for the coefficients of $\frac{\delta^{k-1} q(\xi)}{\delta \xi^{k-1}}$ will be at most limited. Hence $\frac{\delta^{k} \xi^{n}}{\delta \xi^{k}} \simeq 0$ in $\xi=0$. If $k=n$, using the induction hypothesis we find that

$$
\frac{\delta^{n} \xi^{n}}{\delta \xi^{n}}=n(n-1) !+\delta \xi \cdot 0=n ! .
$$

Because $\frac{\delta^{n} \xi^{n}}{\delta \xi^{n}}$ is a constant, $\frac{\delta^{k} \xi^{n}}{\delta \xi^{k}}=0$ for $k>n$.

Lemma 5.11 Let $n \in \mathbb{N}, n \geq 1$ and $a_{1}, \ldots, a_{n} \in \mathbb{R}^{n}$ be standard. Then 


$$
\frac{\delta^{n} c}{\delta \xi^{n}}(0) \simeq \sum_{\substack{k_{1}+2 k_{2}+\ldots+n k_{n}=n \\ k_{1}+k_{2}+\ldots+k_{n}=k}} \frac{k !}{k_{1} ! k_{2} ! \ldots k_{n} !} n ! \prod_{i=1}^{n} a_{i}^{k_{i}}
$$

Proof By (10) and Lemma 5.10 non-infinitesimal contributions to $\frac{\delta^{n} c}{\delta \xi^{n}}(0)$ may occur only for $k_{1}, k_{2}, \ldots, k_{n}$ such that $k_{1}+2 k_{2}+\ldots+n k_{n}=n$; in the latter case the coefficient $\frac{k !}{k_{1} ! k_{2} ! \ldots k_{n} !} \prod_{i=1}^{n} a_{i}^{k_{i}}$ in (10) corresponding to $k \equiv k_{1}+k_{2}+\ldots+k_{n}$ is multiplied by $n !$.

Proposition 5.12 Let $n \in \mathbb{N}$ be standard and let for $k_{1}, \ldots, k_{n}$ with $k_{1}+2 k_{2}+\ldots+n k_{n}=$ $n$ the constants $C_{n, k_{1}, \ldots, k_{n}}$ be given by Lemma 5.9. Then

$$
C_{n, k_{1}, \ldots, k_{n}}=\frac{n !}{k_{1} ! k_{2} ! \ldots k_{n} !} \prod_{i=1}^{n} \frac{1}{i !^{k_{i}}} .
$$

Proof Because the $C\left(n, k_{1}, \ldots, k_{n}\right)$ of Lemma 5.9 do not depend on the choice of the functions, we choose to determine them for the functions $p: \mathbb{X} \rightarrow \mathbb{R}, m: \mathbb{Y} \rightarrow \mathbb{R}$ and $c=m \circ p: \mathbb{X} \rightarrow \mathbb{R}$ as defined in (9). Let $a_{1}, \ldots, a_{n} \in \mathbb{R}^{n}$ be standard. Notice that for $1 \leq i \leq n$ one has $p^{[i]}(0) \simeq i ! a_{i}$. Let $\eta=p(0)=0$. Then, with $k \equiv k_{1}+k_{2}+\ldots+k_{n}$, it holds that $m^{[k]}(\eta)=k$ !. Then it follows from Lemma 5.11 that

$$
\begin{aligned}
c^{[n]}(0) & \simeq \sum_{\substack{k_{1}+2 k_{2}+\ldots+n k_{n}=n \\
k_{1}+k_{2}+\ldots+k_{n}=k}} C_{n, k_{1}, \ldots, k_{n}} m^{\left[k_{1}+k_{2}+\ldots+k_{n}\right]}(0) \prod_{i=1}^{n}\left(p^{[i]}(0)\right)^{k_{i}} \\
& =\sum_{\substack{k_{1}+2 k_{2}+\ldots+n k_{n}=n \\
k_{1}+k_{2}+\ldots+k_{n}=k}} C_{n, k_{1}, \ldots, k_{n}} k ! \prod_{i=1}^{n}\left(i ! a_{i}\right)^{k_{i}} \\
& \simeq \sum_{\substack{k_{1}+2 k_{2}+\ldots+n k_{n}=n \\
k_{1}+k_{2}+\ldots+k_{n}=k}} \frac{k !}{k_{1} ! k_{2} ! \ldots k_{n} !} n ! \prod_{i=1}^{n} a_{i}^{k_{i}} .
\end{aligned}
$$

We define two polynomials $u$ and $v$ in the variables $a_{1}, \ldots, a_{n}$ by

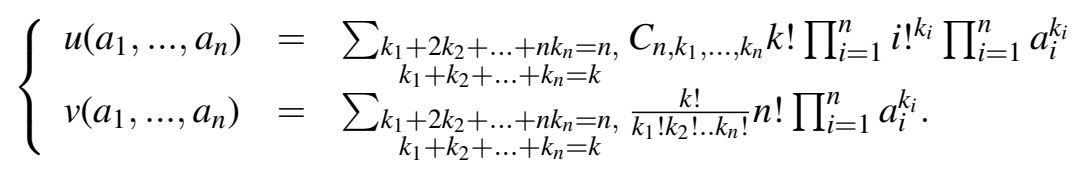

Then $u\left(a_{1}, \ldots, a_{n}\right) \simeq v\left(a_{1}, \ldots, a_{n}\right)$ for all standard $a_{1}, \ldots, a_{n} \in \mathbb{R}$. The polynomial $v$ is clearly standard, and also the polynomial $u$, because the numbers $C_{n, k_{1}, \ldots, k_{n}}$ are 
standard integers. So $u\left(a_{1}, \ldots, a_{n}\right)=v\left(a_{1}, \ldots, a_{n}\right)$ by the Principle of Carnot. Then $u=v$ by Transfer. This means that their coefficients must be equal. This proves the proposition.

Corollary 5.13 (Infinitesimal Faà di Bruno Theorem). Let $n \in \mathbb{N}$ be standard. Let $\phi: \mathbb{X} \rightarrow \mathbb{R}$ and $\psi: \phi(\mathbb{X}) \rightarrow \mathbb{R}$ be functions of class $S^{n}$. Then under the conventions mentioned above, for all $\xi \in[\alpha \cdot \beta]$

$$
\chi^{[n]}(\xi) \simeq \sum_{k_{1}+2 k_{2}+\ldots+n k_{n}=n} \frac{n !}{k_{1} ! k_{2} ! \ldots k_{n} !} \psi^{\left[k_{1}+k_{2}+\ldots+k_{n}\right]}(\phi(\xi)) \prod_{i=1}^{n}\left(\frac{\phi^{[i]}(\xi)}{i !}\right)^{k_{i}} .
$$

\subsection{Continuisation on near-continua of class $S^{n}$}

We prove the transition from the discrete to the continuous in Theorem 5.1 first of all for the case that $n=1$.

Theorem 5.14 Let $\phi:[\alpha \cdots \beta] \rightarrow \mathbb{R}$ be a function of class $S^{1}$ such that $\phi^{[1]}(\xi) \not 0$ for all $\xi \in[\alpha \cdots \beta]$. Let $\psi:[\phi(\alpha) \cdots \phi(\beta)] \rightarrow \mathbb{R}$ be a function of class $S^{1}$. Let $\chi=\psi \circ \phi$. Then $\chi$ is of class $S^{1}$ and for all $\xi \in[\alpha \cdots \beta[$

$$
\chi^{[1]}(\xi)=\psi^{[1]}(\phi(\xi)) \phi^{[1]}(\xi) .
$$

Moreover, let $f={ }^{\circ} \phi, g={ }^{\circ} \psi$ and $h={ }^{\circ} \chi$. Let $a={ }^{\circ} \alpha$ and $b={ }^{\circ} \beta$. Then $g$ is of class $C^{1}$ on $[f(a), f(b)]$ with $g^{\prime}={ }^{\circ}\left(\psi^{[1]}\right)$ and $h$ is of class $C^{1}$ on $[a, b]$, with for all $x \in[a, b]$

$$
h^{\prime}(x)=g^{\prime}(f(x)) f^{\prime}(x)
$$

Proof Formula (11) is the discrete Chain Rule given in (4). By Proposition 5.2 the function $\chi:[\alpha \cdot \beta] \rightarrow \mathbb{R}$ is of class $S^{1}$. By the Theorem of the differentiable shadow the functions $f$ and $h$ are of class $C^{1}$ on $[a, b]$, with $f^{\prime}={ }^{\circ}\left(\phi^{[1]}\right)$ non-zero and $h^{\prime}={ }^{\circ}\left(\chi^{[1]}\right)$. Observe that $f^{-1}$ is of class $C^{1}$ on $[f(a), f(b)]$ with $\left(f^{-1}\right)^{\prime}(y)=1 / f^{\prime}\left(f^{-1}(y)\right) \neq 0$. By the Theorem of the continuous shadow the function $g$ is of class $C^{0}$ on $[f(a), f(b)]$ with $h(x)=g(f(x))$ for all $x \in[a, b]$. Then for all $y \in[f(a), f(b)]$

$$
g(y)=h\left(f^{-1}(y)\right) \text {. }
$$

By the usual Chain Rule $g$ is of class $C^{1}$ on $[f(a), f(b)]$. Then the Chain Rule may also be applied to the composition $h=f \circ g$ and yields (12) for all $x \in[a, b]$.

Let $y \in[f(a), f(b)]$ be standard and $\eta \in[\phi(\alpha) \cdot \phi(\beta)[$ be such that $\eta \simeq y$. Let $\xi \in\left[\alpha \cdots \beta\right.$ [ be such that $\phi(\xi)=\eta$. Put $x=f^{-1}(y)$. Then, applying the Theorem of the 
differentiable shadow to $h$ and $f$, formula (11), the fact that $\psi^{[1]}$ is of class $S^{0}$ and the Theorem of the continuous shadow

$$
g^{\prime}(y)=\frac{h^{\prime}(x)}{f^{\prime}(x)} \simeq \frac{\chi^{[1]}(\xi)}{\phi^{[1]}(\xi)}=\psi^{[1]}(\eta) \simeq\left({ }^{\circ}\left(\psi^{[1]}\right)\right)(y) .
$$

Hence $g^{\prime}(y)=\left({ }^{\circ}\left(\psi^{[1]}\right)\right)(y)$ for all $y \in[f(a), f(b)]$ by the Principle of Carnot and by Transfer, hence $g^{\prime}={ }^{\circ}\left(\psi^{[1]}\right)$.

Next theorem is formulated for arbitrary standard $n \in \mathbb{N}$ and presupposes that the conventions at the beginning of Subsection 5.1 hold.

Theorem 5.15 Let $n \in \mathbb{N}$ be standard. Let $\phi: \mathbb{X} \rightarrow \mathbb{R}$ and $\psi$ : $\phi(\mathbb{X}) \rightarrow \mathbb{R}$ be functions of class $S^{n}$. Let $\xi \in[\alpha \cdot \beta]$. Then $g$ is of class $C^{n}$ on $[f(a), f(b)]$, with $g^{(n)}={ }^{\circ}\left(\psi^{[n]}\right)$, and $h$ is of class $C^{n}$ on $[a, b]$, with for all $x \in[a, b]$

$$
h^{(n)}(x)=\sum_{k_{1}+2 k_{2}+\ldots+n k_{n}=n} \frac{n !}{k_{1} ! k_{2} ! \ldots k_{n} !} g^{\left(k_{1}+k_{2}+\ldots+k_{n}\right)}(f(x)) \prod_{i=1}^{n}\left(\frac{f^{(i)}(x)}{i !}\right)^{k_{i}} .
$$

Proof By external induction. The case $n=1$ is contained in Theorem 5.14. Assume the theorem is proved for $n-1$. We follow, mutatis mutandis, the lines of the proof of Theorem 5.14. We prove first that $g$ is of class $C^{n}$. By Theorem 4.2 the functions $f$ and $h$ are of class $C^{n}$ on $[a, b]$, with $f^{\prime}={ }^{\circ}\left(\phi^{[1]}\right)$ non-zero, $f^{(m)}={ }^{\circ}\left(\phi^{[m]}\right)$ for all $m$ with $2 \leq m \leq n$ and $h^{(n)}={ }^{\circ}\left(\chi^{[n]}\right)$. Observe that $f^{-1}$ is of class $C^{n}$ on $[f(a), f(b)]$ with $\left(f^{-1}\right)^{\prime}(y)=1 / f^{\prime}\left(f^{-1}(y)\right) \neq 0$. By the Theorem of the continuous shadow the function $g$ is of class $C^{0}$ on $[f(a), f(b)]$ with $h(x)=g(f(x))$ for all $x \in[a, b]$. Then for all $y \in[f(a), f(b)]$

$$
g(y)=h\left(f^{-1}(y)\right)
$$

By the ordinary Theorem of Faà di Bruno $g$ is of class $C^{n}$ on $[f(a), f(b)]$. Then the ordinary Theorem of Faà di Bruno holds for the composition $h=f \circ g$ and yields (13).

To prove the remaining part of the theorem, let $\xi \in[\alpha \cdots \beta]$. Then

$$
\chi^{[n]}(\xi) \simeq \sum_{k_{1}+2 k_{2}+\ldots+n k_{n}=n} \frac{n !}{k_{1} ! k_{2} ! \ldots k_{n} !} \psi^{\left[k_{1}+k_{2}+\ldots+k_{n}\right]}(\phi(\xi)) \prod_{i=1}^{n}\left(\frac{\phi^{[i]}(\xi)}{i !}\right)^{k_{i}}
$$

by Proposition 5.13. We put

$$
\tau_{n}(\xi)=\psi^{[n]}(\phi(\xi))\left(\phi^{[1]}(\xi)\right)^{n}
$$


and

$$
\sigma_{n}(\xi)=\sum_{k_{1}+2 k_{2}+\ldots+n k_{n}=n, k_{1}<n} \frac{n !}{k_{1} ! k_{2} ! \ldots k_{n} !} \psi^{\left[k_{1}+k_{2}+\ldots+k_{n}\right]}(\phi(\xi)) \prod_{i=1}^{n}\left(\frac{\phi^{[i]}(\xi)}{i !}\right)^{k_{i}} .
$$

Then

$$
\tau_{n}(\xi) \simeq \chi^{[n]}(\xi)-\sigma_{n}(\xi) .
$$

Now $\tau_{n}(\xi), \chi^{[n]}(\xi)$ and $\sigma_{n}(\xi)$ are of class $S^{0}$. In particular they are limited, and $\phi^{[1]}(\xi)$ is positive appreciable, hence also $\left(\phi^{[1]}(\xi)\right)^{n}$. So for all $\xi \in[\alpha \cdots \beta]$

$$
\psi^{[n]}(\phi(\xi))=\frac{\tau_{n}(\xi)}{\left(\phi^{[1]}(\xi)\right)^{n}} \simeq \frac{\chi^{[n]}(\xi)-\sigma_{n}(\xi)}{\left(\phi^{[1]}(\xi)\right)^{n}} .
$$

We put $t_{n}={ }^{\circ} \tau_{n}$ and $s_{n}={ }^{\circ} \sigma_{n}$. Then $t_{n}, s_{n}:[a, b] \rightarrow \mathbb{R}$ are continuous. Notice that $k_{1}+2 k_{2}+\ldots+n k_{n}=n, k_{1}<n$ implies that $k_{1}+k_{2}+\ldots+k_{n}<n$, so ${ }^{\circ}\left(\psi^{\left[k_{1}+k_{2}+\ldots+k_{n}\right]}\right)=g^{\left(k_{1}+k_{2}+\ldots+k_{n}\right)}$ by the induction hypothesis. Hence $s_{n}$ satisfies

$$
s_{n}(x)=\sum_{k_{1}+2 k_{2}+\ldots+n k_{n}=n, k_{1}<n} \frac{n !}{k_{1} ! k_{2} ! \ldots k_{n} !} g^{\left(k_{1}+k_{2}+\ldots+k_{n}\right)}(f(x)) \prod_{i=1}^{n}\left(\frac{f^{(i)}(x)}{i !}\right)^{k_{i}} .
$$

for all $x \in[a, b]$. Then it follows from (14) and (13) that for all $x \in[a, b]$

$$
t_{n}(x)=h^{(n)}(x)-s_{n}(x)=g^{(n)}(f(x))\left(f^{\prime}(x)\right)^{n} .
$$

Let $y \in[f(a), f(b)]$ be standard and $\eta \in[\phi(\alpha) \cdot \phi(\beta)]$ be such that $\eta \simeq y$. Let $\xi \in[\alpha \cdots \beta]$ be such that $\phi(\xi)=\eta$. Put $x=f^{-1}(y)$. Then it follows from the previous calculations, the Theorem of the differentiable shadow, the fact that $\psi^{[n]}$ is of class $S^{0}$ and the Theorem of the continuous shadow that

$$
g^{(n)}(y)=\frac{t_{n}(x)}{\left(f^{\prime}(x)\right)^{n}} \simeq \frac{t_{n}(\xi)}{\left(\phi^{[1]}(\xi)\right)^{n}}=\psi^{[n]}(\eta) \simeq{ }^{\circ}\left(\psi^{[n]}\right)(y) .
$$

Hence $g^{(n)}(y)=\left({ }^{\circ}\left(\psi^{[n]}\right)\right)(y)$ for all $y \in[f(a), f(b)]$ by the Principle of Carnot and by Transfer, hence $g^{(n)}=\left({ }^{\circ}\left(\psi^{[n]}\right)\right)$.

The general Theorem 5.1 follows from two observations made beforehand. Firstly, the condition that $\phi^{[1]}(\bar{\xi}) \not 0$ at some limited point $\bar{\xi} \in \mathbb{X}$ implies the existence of a near-interval $[\alpha \cdots \beta]$ with $\alpha, \beta \in \mathbb{X}$ limited and $\alpha \succsim \bar{\xi} \succsim \beta$ such that $\phi^{[1]}(\xi) \not 0$ for all $\xi \in[\alpha \cdot \beta]$. Secondly, the condition of $\phi^{[1]}(\xi) \gtrsim 0$ to hold on $[\alpha \cdots \beta]$ was adopted during the previous discourse by mere convenience, and the proof easily carries over to the case where $\phi^{[1]}(\xi) \succsim 0$ on $[\alpha \cdots \beta]$. 


\section{Nearly-continuous properties of discrete functions of two variables}

We consider discrete functions defined on Cartesian products of two nearcontinua $\mathbb{X}, \mathbb{Y} \subset \mathbb{R}$. We denote the difference of two successive points of the set $\mathbb{X}$ by $\delta x$ and the difference of two successive points of the set $\mathbb{Y}$ by $\delta y$. The quantities $\delta x$ and $\delta y$ may or may not be interrelated and may or may not be constant. We extend the notion of functions of class $S^{n}$ to functions of two variables. Then we consider the transition from the discrete to the continuous for such functions.

Definition 6.1 Let $A \subseteq \mathbb{R}^{2}$ and $f: A \rightarrow \mathbb{R}$. Let $\left(x_{1}, x_{2}\right) \in A$. The function $f$ is said to be $S$-continuous at $\left(x_{1}, x_{2}\right)$ if for all $\left(y_{1}, y_{2}\right) \in A$

$$
x_{1} \simeq y_{1}, x_{2} \simeq y_{2} \Rightarrow f\left(x_{1}, x_{2}\right) \simeq f\left(y_{1}, y_{2}\right) .
$$

The function $f$ is said to be $S$-continuous on $A$ if $f$ is $S$-continuous at all $\left(x_{1}, x_{2}\right) \in A$.

For convenience we consider from now one functions defined on the whole Cartesian product $\mathbb{X} \times \mathbb{Y}$.

Definition 6.2 Let $f: \mathbb{X} \times \mathbb{Y} \rightarrow \mathbb{R}$. The function $f$ is said to be of class $S^{00}$ if $f$ is limited and $S$-continuous at every limited $(x, y) \in \mathbb{X} \times \mathbb{Y}$.

Just as standard everywhere continuous real functions of one variable are of class $S^{0}$, one may show that standard everywhere continuous real functions of two variables are of class $S^{00}$.

Definition 6.3 Let $m, n \in \mathbb{N}$ and $f: \mathbb{X} \times \mathbb{Y} \rightarrow \mathbb{R}$. We note the $m^{\text {th }}$ difference quotient with respect to the first variable by $f_{1}^{[m]}$ and the $n^{\text {th }}$ difference quotient with respect to the second variable by $f_{2}^{[n]}$.

We may denote the $m^{\text {th }}$ difference quotient with respect to the first variable of the $n^{\text {th }}$ difference quotient with respect to the second variable of $f$ by $f_{12}^{[m][n]}$ (equal to the $n^{\text {th }}$ difference quotient with respect to the second variable of the $m^{\text {th }}$ difference quotient with respect to the first variable, i.e. to $f_{21}^{[n][m]}$ ). We identify $f_{1}^{[0]}, f_{2}^{[0]}$ and $f_{12}^{[0][0]}$ with $f$. A function $f: \mathbb{R}^{2} \rightarrow \mathbb{R}$ is of class $C^{m n}$ if all mixed partial derivatives up to order $m$ in the first variable and up to order $n$ in the second variable exist and are continuous in both variables. A function is of class $C^{k}$ if the mixed partial derivatives $\frac{\partial^{m} \partial^{n} f}{\partial x^{m} \partial y^{n}}$ exist and are continuous in both variables for all $m, n$ with $m, n \leq k$. We define by analogy functions of class $S^{m n}$ and $S^{k}$ for standard $m, n$ and $k$. 
Definition 6.4 Let $m, n \in \mathbb{N}$ be standard and $f: \mathbb{X} \times \mathbb{Y} \rightarrow \mathbb{R}$. We say that $f$ is of class $S^{m n}$ if $f_{12}^{[i][j]}$ is of class $S^{00}$ for all $i, j$ with $0 \leq i \leq m$ and $0 \leq j \leq n$.

Definition 6.5 Let $k \in \mathbb{N}$ be standard and $f: \mathbb{X} \times \mathbb{Y} \rightarrow \mathbb{R}$. The function $f$ is said to be of class $S^{k}$ if the difference quotients $f_{12}^{[m][n]}$ are of class $S^{00}$ for all $m, n \in \mathbb{N}$ such that $m, n \leq k$.

The next proposition is an immediate consequence of Definition 6.4.

Proposition 6.6 Let $k \in \mathbb{N}$ be standard and $f: \mathbb{X} \times \mathbb{Y} \rightarrow \mathbb{R}$ be of class $S^{k}$. Let $m, n \in \mathbb{N}$ be such that $m, n \leq k$. Then $f_{1}^{[m]}$ is of class $S^{0 n}$ and $f_{2}^{[n]}$ is of class $S^{m 0}$.

Next theorem relates functions of class $S^{m n}$ to functions of class $C^{n m}$, and, more in general, functions of two-variables of class $S^{k}$ to functions of class $C^{k}$.

Theorem 6.7 Let $k \in \mathbb{N}$ be standard and $\mathbb{X}$ and $\mathbb{Y}$ be near-continua of class $S^{k}$. Let $f: \mathbb{X} \times \mathbb{Y} \rightarrow \mathbb{R}$ be of class $S^{k}$. Then ${ }^{\circ} f$ is of class $C^{k}$ on $\mathbb{R}^{2}$ and for all $m, n \leq k$

$$
{ }^{\circ}\left(f_{12}^{[m][n]}\right)=\frac{\partial^{m} \partial^{n}\left({ }^{\circ} f\right)}{\partial x^{m} \partial y^{n}} .
$$

In order to prove this theorem, we start by recalling two results from the literature.

Theorem 6.8 (Theorem of the continuous shadow, two variables) Let $f: \mathbb{X} \times \mathbb{Y} \rightarrow \mathbb{R}$ be of class $S^{00}$. Then there exists a unique standard function ${ }^{\circ} f: \mathbb{R}^{2} \rightarrow \mathbb{R}$ such that $f(x, y) \simeq{ }^{\circ} f(x, y)$ for all limited $(x, y) \in \mathbb{X} \times \mathbb{Y}$. In fact $f$ is continuous on $\mathbb{R}^{2}$.

The theorem is essentially a consequence of a general theorem on metric spaces, which can be found in several textbooks, like [8].

Proposition 6.9 Let $\mathbb{X}$ be a near-continuum of class $S^{1}$ and $\mathbb{Y}$ be a near-continuum of class $S^{0}$. If $f: \mathbb{X} \times \mathbb{Y} \rightarrow \mathbb{R}$ is of class $S^{10}$, its shadow ${ }^{\circ} f: \mathbb{R}^{2} \rightarrow \mathbb{R}$ is of class $C^{10}$ and one has

$$
{ }^{\circ}\left(f_{1}^{[1]}\right)=\frac{\partial^{\circ} f}{\partial x} .
$$

The proposition has been proved in [4] for functions defined on Cartesian products of equidistant near-continua. Due to Theorem 5.14 there is no difficulty extending the proof to the above case.

We extend Proposition 6.9 to higher order. 
Proposition 6.10 Let $m \in \mathbb{N}$ be standard. Let $\mathbb{X}$ be a near-continuum of class $S^{m}$ and $\mathbb{Y}$ be a near-continuum of class $S^{0}$. If $f: \mathbb{X} \times \mathbb{Y} \rightarrow \mathbb{R}$ is of class $S^{m 0}$, its shadow ${ }^{\circ} f: \mathbb{R}^{2} \rightarrow \mathbb{R}$ is of class $C^{m 0}$ and one has for all $i$ with $0 \leq i \leq m$

$$
{ }^{\circ}\left(f_{1}^{[i]}\right)=\frac{\partial^{i}\left({ }^{\circ} f\right)}{\partial x^{i}}
$$

Proof By external induction in $i$. For $i=0$ nothing has to be proved and the case $i=1$ is contained in formula (15). Assume the equality holds for some standard $i<m$. By definition $f_{1}^{[i]}$ and $\left(f_{1}^{[i]}\right)_{1}^{[1]}=f_{1}^{[i+1]}$ are of class $S^{00}$. Then $f_{1}^{[i]}$ is of class $S^{10}$. Hence by formula (15) and the induction hypothesis

$$
{ }^{\circ}\left(f_{1}^{[i+1]}\right)={ }^{\circ}\left(\left(f_{1}^{[i]}\right)_{1}^{[1]}\right)=\frac{\partial}{\partial x}^{\circ}\left(f_{1}^{[i]}\right)=\frac{\partial}{\partial x}\left(\frac{\partial^{i}\left({ }^{\circ} f\right)}{\partial x^{i}}\right)=\frac{\partial^{i+1}\left({ }^{\circ} f\right)}{\partial x^{i+1}} .
$$

Let $n \in \mathbb{N}$ be standard. By analogy, if $\mathbb{X}$ is a near-continuum of class $S^{0}$ and $\mathbb{Y}$ is a near-continuum of class $S^{n}$, and $f: \mathbb{X} \times \mathbb{Y} \rightarrow \mathbb{R}$ is of class $S^{0 n}$, its shadow ${ }^{\circ} f: \mathbb{R}^{2} \rightarrow \mathbb{R}$ is of class $C^{0 n}$ and one has for all $j$ with $0 \leq j \leq n$

$$
\circ\left(f_{2}^{[j]}\right)=\frac{\partial^{j}\left({ }^{\circ} f\right)}{\partial y^{j}} \text {. }
$$

Proof of Theorem 6.7: Let $m, n \in \mathbb{N}$, with $m, n \leq k$. By Proposition 6.6 the difference quotient $f_{2}^{[n]}$ is of class $S^{m 0}$ and by definition $f$ is of class $S^{0 n}$. Then by Proposition 6.10 and (17)

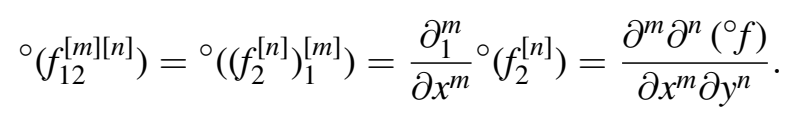

\section{A higher order DeMoivre-Laplace Theorem}

As an application we consider a problem of continuisation of higher order in two dimensions. We extend the DeMoivre-Laplace Theorem on the transition of the binomial probability distribution $B(N, j)$ to the Gaussian distribution $G(t, x)=\frac{\exp \left(-x^{2} /(2 t)\right)}{\sqrt{2 \pi t}}$ to their successive difference quotients respectively partial derivatives.

Firstly we recall a nonstandard version of the common DeMoivre-Laplace Theorem, such as presented in $[5, \mathrm{p} .2]$. It states that a rescaling $b(t, x)$ of the of the Pascal Triangle corresponding to the binomial probability distribution $B(N, j)$ is nearly equal to the Gaussian function $G(t, x)$. The rescaling of the variables $N$ and $j$ corresponds to a 
change of scale of type centralization-reduction in two dimensions, into the so-called binomial cone. This cone can be seen as the union of two two-dimensional grids, equally spaced in each dimension. Then we will be able to apply the techniques of the previous sections to continuisations on near-continua, and obtain a DeMoivre-Laplace Theorem of higher order: all partial difference quotients of standard order of $b(t, x)$ are nearly equal to the corresponding partial derivatives of $G(t, x)$.

The case of first order in time and second order in space has been proved in [4], using a general method of transition from partial difference equations to partial differential equations of first and second order. With respect the general case of transitions at any standard order we present here a streamlined version of material contained in [11] and [6].

In Subsection 7.1 we effectuate the rescaling of the set of binomial coefficients, recall the nonstandard version of the DeMoivre-Laplace Theorem of [5] and state the higher order DeMoivre-Laplace Theorem. We also discuss the relation of the theorems to some nonstandard approaches to stochastic processes and the heat equation. The higher order DeMoivre-Laplace Theorem will be proved using some partial difference equations for the rescaled binomial coefficients. These will be presented in Subsection 7.2. The transition to continuity towards the higher order DeMoivre-Laplace Theorem will be shown in Subsection 7.3.

\subsection{Rescaling of the binomial coefficients}

Definition 7.1 Let $\delta t>0$. We define the binomial cone $C_{\delta t}$ by

$$
C_{\delta t}=\left\{(t, x) \in \mathbb{R}^{2}|t \geqslant 0,| x \mid \leqslant t / \sqrt{\delta t}, \exists \nu, j \in \mathbb{N}, t=\nu \delta t, x=(2 j-\nu) \sqrt{\delta t}\right\} .
$$

We write

$$
\left\{\begin{array}{l}
\mathbb{T}=\{\nu \delta t \mid \nu \in \mathbb{N}\} \\
\delta x=2 \sqrt{\delta t}
\end{array}\right.
$$

If $\delta t \simeq 0$ the upper and lower boundaries of $C_{\delta t}$ are nearly vertical "discrete lines" with infinitely large slope $\pm 1 / \sqrt{\delta t}$. This implies that $C_{\delta t}$ contains all points $(t, x)$ such that $t$ is appreciable and multiple of $\delta t$, and $x$ is limited and of the form $x=(2 j-\nu) \sqrt{\delta t}$ for some $\nu \in \mathbb{N}$ and $j \in \mathbb{Z}$.

For convenience we assume that $\frac{1}{\delta t}$ is an integer. 
Definition 7.2 Let $(t, x) \in C_{\delta t}$, We write

$$
\begin{aligned}
\nu_{t} & =\frac{t}{\delta t} \\
j_{t, x} & =\frac{t}{2 \delta t}+\frac{x}{\delta x} .
\end{aligned}
$$

Conversely, if $\nu \in \mathbb{N}$, and $j \in \mathbb{N}$ is such that $0 \leq j \leq \nu$, we write

$$
\begin{aligned}
t_{\nu} & =\nu \delta t \\
x_{\nu, j} & =(j-\nu / 2) \delta x .
\end{aligned}
$$

Definition 7.3 Let $\nu, j \in \mathbb{N}$ be such that $0 \leq j \leq \nu$. Then we write

$$
B(\nu, j)=\left(\begin{array}{l}
\nu \\
j
\end{array}\right)\left(\frac{1}{2}\right)^{\nu} \text {. }
$$

Definition 7.4 The binomial function $b: C_{\delta t} \rightarrow \mathbb{R}$ is defined by

$$
b(t, x)=\frac{1}{\delta x} B\left(\nu_{t}, j_{t, x}\right) .
$$

With respect to space we normalized and centralized around the mean. In particular if $x=1$, we reduced the distance with respect to the mean by the standard deviation. If $\nu$ is infinitely large, a set of positive integers is transformed into an infinitely fine sequence of points equally spaced at a distance of $x=2 / \sqrt{\nu}$, and symmetric with respect to the origin. With respect to time we reduce the increments to $\delta t=1 / \nu$. Observe that the binomial cone $C_{\delta t}$ is a subset of the union of two Cartesian products with horizontal step $2 \delta t$ and vertical step $\delta x=2 \sqrt{\delta t}$. So the notations and results on difference quotients of Section 6 will be applied to Cartesian products of equidistant near-continua, up to adapting some technical details.

Within this setting it is possible to state a DeMoivre-Laplace Theorem in the form of a near-equality of the rescaled binomial coefficients represented by the function $b(t, x)$ and the Gaussian function $G(t, x)$.

Theorem 7.5 (DeMoivre-Laplace theorem, nonstandard) Let $\delta t \simeq 0$. Then for all $(t, x) \in C_{\delta t}$ such that $t$ is appreciable and $x$ is limited,

$$
b(t, x) \simeq \frac{1}{\sqrt{2 \pi t}} \exp \left(-\frac{x^{2}}{2 t}\right) .
$$

For a proof we refer to $[5$, p. 19$]$.

There exists a natural relation between the nonstandard DeMoivre-Laplace Theorem and the discrete nonstandard stochastic processes which where first proposed by Anderson in 
[2] as an alternative for continuous-time stochastic processes. In this context stochastic processes typically have infinitesimal increments for infinitesimal time-increments $\delta t$. The fundamental process is the Wiener Walk $W_{t}$, which satisfies $W_{0}=0$ and is such that its increments $\delta W_{t}= \pm \sqrt{\delta t}$ are independent and equiprobable. The nonstandard DeMoivre-Laplace Theorem expresses the near-equality of the "discrete density function" $b(t, x)$ related to the Wiener Walk and the usual Gaussian function which is the density function related to Brownian Motion. Indeed, it is not difficult to see that for all $(t, x) \in C_{\delta t}$

$$
\operatorname{Pr}\left\{W_{t}=x\right\}=b(t, x) \delta x .
$$

Anderson's approach lead to general methods of interplay between stochastic processes in discrete time and continuous time based on Loeb-measure, introduced in [13]. Still in the context of discrete stochastic processes, general conditions in terms of nearequivalence have been given for the Standard Normal Distribution to hold approximately. Indeed, in the last chapter in [15] and in [3] it is shown that a large class of nonstandard discrete stochastic processes are nearly equivalent to the Wiener Walk. One of this conditions is of Lindeberg type. Nonstandard Lindeberg conditions are used also by Weisshaupt [17], to show near-equality of solutions - which are expectations for stochastic processes - of a discrete and a continuous heat equation.

The following proposition is a first consequence of Theorem 7.5.

Proposition 7.6 Let $(t, x) \in C_{\delta t}$ be such that $t$ is fixed and appreciable and $x$ is limited. Let $n \in \mathbb{N}$ be standard and arbitrary. Then

(1) Let $t$ be fixed and appreciable. Then $b(t, \cdot)$ is of class $S^{0}$.

(2) Let $x$ be fixed and limited. Then $b(\cdot, x)$ is of class $S^{0}$ on every set $I=$ $\left\{t \in \mathbb{T} \mid t_{0} \leq t\right\}$ with $t_{0}$ appreciable.

We will extend Proposition 7.6 to higher order.

Theorem 7.7 Let $k \in \mathbb{N}$ be standard. Let $t_{0} \gtrsim 0$ be limited and $D=\{(t, x) \in$ $\left.C_{\delta t} \mid t \geq t_{0}\right\}$. Then $b$ is of class $S^{k}$ on $D$.

From this theorem we derive the near-equality of difference quotients of the binomial function and the Gaussian function at all standard orders.

Theorem 7.8 (Higher order DeMoivre-Laplace theorem) Let $\delta t \simeq 0$. For every standard $m, n \in \mathbb{N}$ it holds that for all $(t, x) \in C_{\delta t}$ such that $t$ is appreciable and $x$ is limited

$$
b_{12}^{[m][n]}(t, x) \simeq \frac{\partial^{m} \partial^{n} G(t, x)}{\partial t^{m} \partial x^{n}} .
$$


The proofs of Theorem 7.7 and Theorem 7.8 use some convenient ordinary and partial difference equations for the binomial function, which will be derived from combinatorial properties of the binomial coefficients.

\subsection{Difference equations for the binomial function}

The first lemma states a first-order difference equation with respect to the space-variable $x$.

Lemma 7.9 For all $(t, x) \in C_{\delta t}$ such that $t>0, x<\frac{t}{\sqrt{\delta t}}$

$$
b_{2}^{[1]}(t, x)=-b(t, x) \frac{x+\frac{1}{2} \delta x}{t+\frac{x}{2} \delta x+\frac{1}{2} \delta x^{2}} .
$$

The lemma is an easy consequence of the combinatorial formula

$$
B(\nu, j+1)=B(\nu, j) \cdot \frac{\nu-j}{j+1} .
$$

We derive now a partial difference equation for the binomial function, which is a discrete version of the heat equation.

Proposition 7.10 For all $(t, x) \in C_{\delta t}$

$$
b_{1}^{[1]}(t, x)=\frac{1}{2} b_{2}^{[2]}(t, x-\delta x) .
$$

Proof From the Pascal Triangle one derives

$$
B\left(\nu_{t}+1, j_{t, x}+1\right)=\frac{1}{2} B\left(\nu_{t}, j_{t, x}\right)+\frac{1}{2} B\left(\nu_{t}, j_{t, x}+1\right) .
$$

Then by Definition 7.2

$$
b(t+\delta t, x)=\frac{1}{2} b(t, x+\sqrt{\delta t})+\frac{1}{2} b(t, x-\sqrt{\delta t}) .
$$

Repeating this step, one obtains

$$
b(t+2 \delta t, x)=\frac{1}{4} b(t, x+\delta x)+\frac{1}{2} b(t, x)+\frac{1}{4} b(t, x-\delta x) .
$$

Hence

$$
b(t+2 \delta t, x)-b(t, x)=\frac{1}{4} b(t, x+\delta x)-\frac{1}{2} b(t, x)+\frac{1}{4} b(t, x-\delta x) .
$$

This implies (20). 
The discrete heat equation (20) extends to higher order.

Theorem 7.11 For all $(t, x) \in C_{\delta t}$ and all $m \in \mathbb{N}$ one has, as long as $-\frac{t}{\sqrt{\delta t}} \leq$ $x-m \delta x, x+m \delta x \leq \frac{t}{\sqrt{\delta t}}$

$$
b_{1}^{[m]}(t, x)=\frac{1}{2^{m}} b_{2}^{[2 m]}(t, x-m \delta x) .
$$

Proof By induction in $m$. Assume (21) holds. Then

$$
\begin{aligned}
b_{1}^{[m+1]}(t, x) & =\left(b_{1}^{[m]}\right)_{1}^{[1]}(t, x)=\frac{b_{1}^{[m]}(t+2 \delta t, x)-b_{1}^{[m]}(t, x)}{2 \delta t} \\
& =\frac{\frac{1}{2^{m}} b_{2}^{[2 m]}(t+2 \delta t, x-m \delta x)-\frac{1}{2^{m}} b_{2}^{[2 m]}(t, x-m \delta x)}{2 \delta t} . \\
& =\frac{1}{2^{m}}\left(b_{2}^{[2 m]}\right)_{1}^{[1]}(t, x-m \delta x)=\frac{1}{2^{m}} \frac{1}{2}\left(b_{2}^{[2 m]}\right)_{2}^{[2]}(t, x-(m+1) \delta x) \\
& =\frac{1}{2^{m+1}} b_{2}^{[2(m+1)]}(t, x-(m+1) \delta x) .
\end{aligned}
$$

\subsection{Proof of the higher order DeMoivre-Laplace theorem}

Theorem 7.11 reduces difference quotients of the binomial function with respect to time to difference quotients with respect to space (of even order). So regularity properties of difference quotients with respect to time may be derived from corresponding properties of difference quotients with respect to space.

The first-order difference equation with respect to space established in Section 7.2 enables to prove that the binomial function is of class $S^{n}$ in the space-variable for all standard $n$. Then the higher order difference quotients of the binomial function with respect to space are nearly equal to the corresponding higher order differential quotients of the Gaussian function, given explicitly in [1]. The higher-order heat difference equation of Theorem 7.11 will enable to conclude the proof.

Lemma 7.12 Let $(t, x) \in C_{\delta t}$ be such that $t$ is fixed and appreciable and $x$ is limited. Let $n \in \mathbb{N}$ be standard and arbitrary. Then

(1) The function $b_{2}^{[n]}(t, \cdot)$ is of class $S^{0}$. 
(2)

$$
\text { (22) } \begin{aligned}
b_{2}^{[n]}(t, x) & \simeq \frac{\partial^{n} G(t, x)}{\partial x^{n}} \\
= & (-1)^{n} \frac{n !}{\sqrt{2 \pi t^{n+1}}} e^{-\frac{x^{2}}{2 t} \sum_{k=0}^{[n / 2]}(-1)^{k} \frac{1}{k ! 2^{k}(n-2 k) !}\left(\frac{x}{\sqrt{t}}\right)^{n-2 k} .}
\end{aligned}
$$

(3) The function $b$ is of class $S^{0 n}$ on every set $D=\left\{(t, x) \in C_{\delta t} \mid t \geq t_{0}\right\}$ with $t_{0} \gtrsim 0$.

Proof We use the difference equation with respect to $x$ given by (19), rewritten to

$$
b_{2}^{[1]}(t, x)=b(t, x)\left(-\frac{x+\frac{1}{2} \delta x}{t+\frac{x}{2} \delta x+\frac{1}{2} \delta x^{2}}\right) .
$$

We put

$$
c(t, x)=-\frac{x+\frac{1}{2} \delta x}{t+\frac{x}{2} \delta x+\frac{1}{2} \delta x^{2}} .
$$

Notice that, as long as $t$ is appreciable, the function $c(t, \cdot)$, being a rational function of polynomials with limited coefficients without limited poles, is of class $S^{k}$ for all standard $k$.

(1) By external induction. The function $b(t, \cdot)$ is of class $S^{0}$ by Proposition 7.6.1. Let $n \in \mathbb{N}$ be standard and assume $b(t, \cdot)$ is of class $S^{n}$. Because $c(t, \cdot)$ is also of class $S^{n}$, the product $b(t, \cdot) c(t, \cdot)$ is of class $S^{n}$ by Proposition 3.9. So $b_{2}^{[1]}(t, \cdot)$ is of class $S^{n}$. Again by Proposition 7.6.1, and by the Lemma of the discrete integral $b(t, \cdot)$ is of class $S^{n+1}$.

(2) It follows from Theorem 6.7 that the near-equality $\frac{\delta_{2}^{n} b}{\delta x^{n}}(t, x) \simeq \frac{\partial^{n} G(t, x)}{\partial x^{n}}$ is valid for all limited $x$ such that $(t, x) \in C_{\delta t}$. It is known [1] that

$$
\frac{\partial^{n} G(t, x)}{\partial x^{n}}=(-1)^{n} \frac{n !}{\sqrt{2 \pi t^{n+1}}} e^{-\frac{x^{2}}{2 t}} \sum_{k=0}^{[n / 2]}(-1)^{k} \frac{1}{k ! 2^{k}(n-2 k) !}\left(\frac{x}{\sqrt{t}}\right)^{n-2 k} .
$$

(One may show this by applying the formula of Faà di Bruno to $\exp \left(-x^{2} /(2 t)\right)$, with $k_{2}=k$ and $k_{1}=n-2 k$, noting that $\left.k_{3}=\cdots=k_{n}=0\right)$. This implies (22).

(3) Let $t_{0} \gtrsim 0$ and $D=\left\{(t, x) \in C_{\delta t} \mid t \geq t_{0}\right\}$. By Proposition 7.6 the function $b$ is of class $S^{00}$ on $D$. Secondly $b_{2}^{[n]}(t, x) \simeq \frac{\partial^{n} G(t, x)}{\partial x^{n}}$ and $\frac{\partial^{n} G(t, x)}{\partial x^{n}}$ is of class $S^{00}$ on $D$. So $b_{2}^{[n]}$ is also of class $S^{00}$ on $D$. Hence $b$ is of class $S^{0 n}$ on $D$. 
Proof of Theorem 7.7. Let $m, n \in \mathbb{N}$ be such that $m, n \leq k$. Let $t_{0} \gtrsim 0$ be limited and $(t, x) \in C_{\delta t}$ be such that $t \geq t_{0}$. Note that, whenever defined,

$$
b_{12}^{[m][n]}(t, x)=\frac{1}{2^{m}} b_{2}^{[n+2 m]}(t, x-2 m \delta x) .
$$

By Lemma 7.12 the function $b_{2}^{[n+2 m]}$ is of class $S^{00}$ on $D$, hence $b_{12}^{[m][n]}$ is also of class $S^{00}$ on $D$. We conclude that $b$ is of class $S^{k}$ on $D$.

Proof of the higher order DeMoivre-Laplace Theorem. Let $m, n \in \mathbb{N}$ be standard and $(t, x) \in C_{\delta t}$ be such that $t$ is appreciable and $x$ is limited. Let $t_{0}$ be standard with $0<t_{0}<t$. By Theorem 7.7 the binomial function $b$ is of class $S^{m n}$ on $D \equiv\left\{(t, x) \in C_{\delta t} \mid t \geq t_{0}\right\}$. Now ${ }^{\circ}\left(b_{\mid D}\right)=G_{\mid\left[t_{0}, \infty\right) \times \mathbb{R}}$ by Theorem 7.5. Then by Theorem 6.7 for every limited $(t, x) \in D$

$$
b_{12}^{[m][n]}(t, x) \simeq \frac{\partial^{m} \partial^{n} G(t, x)}{\partial t^{m} \partial x^{n}}
$$

The properties of regularity of higher order presented in this article may suggest to study some problems related to partial differential equations by means of the corresponding partial difference equations with infinitesimal steps. Partial difference equations can sometimes be seen as more natural, when partial differential equations are continuous "idealisations" of discrete physical problems. Also, partial difference equations may be studied within infinitesimal discretisations of real space, instead of within functional spaces which often are needed for the study of partial differential equations. As for the particular example of the present section, the binomial function is a solution of a discrete heat equation with on the boundary a "discrete Dirac function" (a nonstandard function $\Delta(x)$ which satisfies $\Delta(0)=1 / \delta x$ and is zero for multiples of $\delta x)$. The higher order DeMoivre-Laplace Theorem could then be used to study continuous properties

of solutions of the discrete heat equation or continuisations of them, given boundary conditions of various types and even in the case of free boundaries. The nonstandard Faà di Bruno Theorem can be of some value for more general parabolic partial difference equations, which may very well involve non-equidistant grids.

Acknowledgement The author thanks the referees for valuable suggestions for improvement.

\section{References}

[1] M. Abramowitz and I.A. Stegun, Handbook of Mathematical Functions, Dover, NewYork, 1965. 
[2] R.M. Anderson, A nonstandard representation for Brownian motion and Itô integration, Israel Journal of Mathematics 25 (1976), 15-46; doi: 10.1007/BF02756559.

[3] E. Benoît, Random walks and stochastic differential equations, in: F. and M. Diener (eds.), Nonstandard Analysis in Practice, Springer Universitext, 1995, 71-90.

[4] I.P. van den Berg, On the relation between elementary partial difference equations and partial differential equations, Ann. Pure App. Logic 92 (1998), 235-265; doi: 10.1016/S0168-0072(98)00006-2.

[5] I.P. van den Berg, Principles of Infinitesimal Stochastic and Financial Analysis, World Scientific, 2000.

[6] I.P. van den Berg, A DeMoivre-Laplace theorem of all orders of regularity, in: Communications of the Laufen Colloquium on Science 2007, A. Ruffing, A. Suhrer, J. Suhrer (Eds.), Shaker Publishing, Maastricht/Aachen, 2007, 335-360.

[7] F. Diener and M. Diener (eds.), Nonstandard Analysis in Practice, Universitext, Springer 1995.

[8] F. Diener and G. Reeb, Analyse Non Standard, Hermann, Paris, 1989.

[9] F. Diener, Cours d'Analyse Non Standard, Office des Publ. Univ. Alger, 1983.

[10] W. Feller, An Introduction to Probability Theory and its Applications, Vol. 1, $3^{\text {rd }}$ ed., Wiley, 1968.

[11] H. Gião, Um Teorema de DeMoivre-Laplace de Ordem arbitrária, Masters Thesis, University of Évora, Portugal, 2005.

[12] S.G. Krantz and H.R. Parks, A Primer of Real Analytic Functions, $2^{\text {nd }}$ ed., Birkhäuser, 2002.

[13] P.A. Loeb, Conversion from nonstandard to standard measure spaces and applications in probability theory, Trans. Amer. Math. Soc. 211 (1975), 113-122; doi: 10.1090/S00029947-1975-0390154-8.

[14] E. Nelson, Internal set theory, Bull. Amer. Math. Soc. 83(6)(1977), 1165-1198; doi:10.1090/S0002-9904-1977-14398-X.

[15] E. Nelson, Radically Elementary Probability Theory, Princ. Univ. Press, 1987.

[16] A. Robinson, Non-standard Analysis, $3^{\text {rd }}$ ed., Princ. Univ. Press, 1996.

[17] H. Weisshaupt, Diffusion processes via parabolic equations: an infinitesimal approach to Lindeberg's limit theorem, J. Log. Anal. 1:2 (2009), 1-29; doi: 10.4115/jla.2009.1.2.

University of Évora,, Department of Mathematics,, Portugal

ivdb@uevora.pt

Received: 13 September 2012 Revised: 21 May 2013 